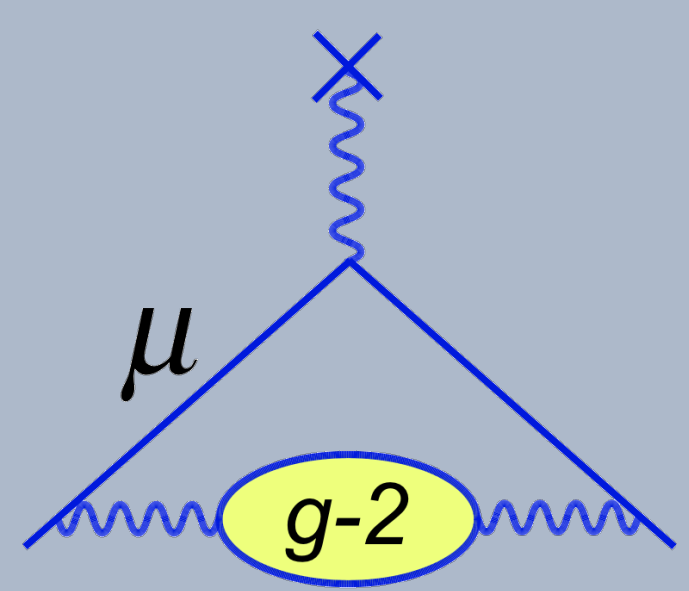

\title{
First Results From the Fermilab Muon $g-2$ Experiment
}

\section{Eremey Valetov}

Michigan State University

On behalf of the Muon $g-2$ Collaboration

\section{$\frac{\text { MICHIGAN STATE }}{\text { U N I V E R S I T Y P.S. DEPARTMENT OF }} \begin{aligned} & \text { Office of } \\ & \text { Science }\end{aligned}$}




\section{Introduction}
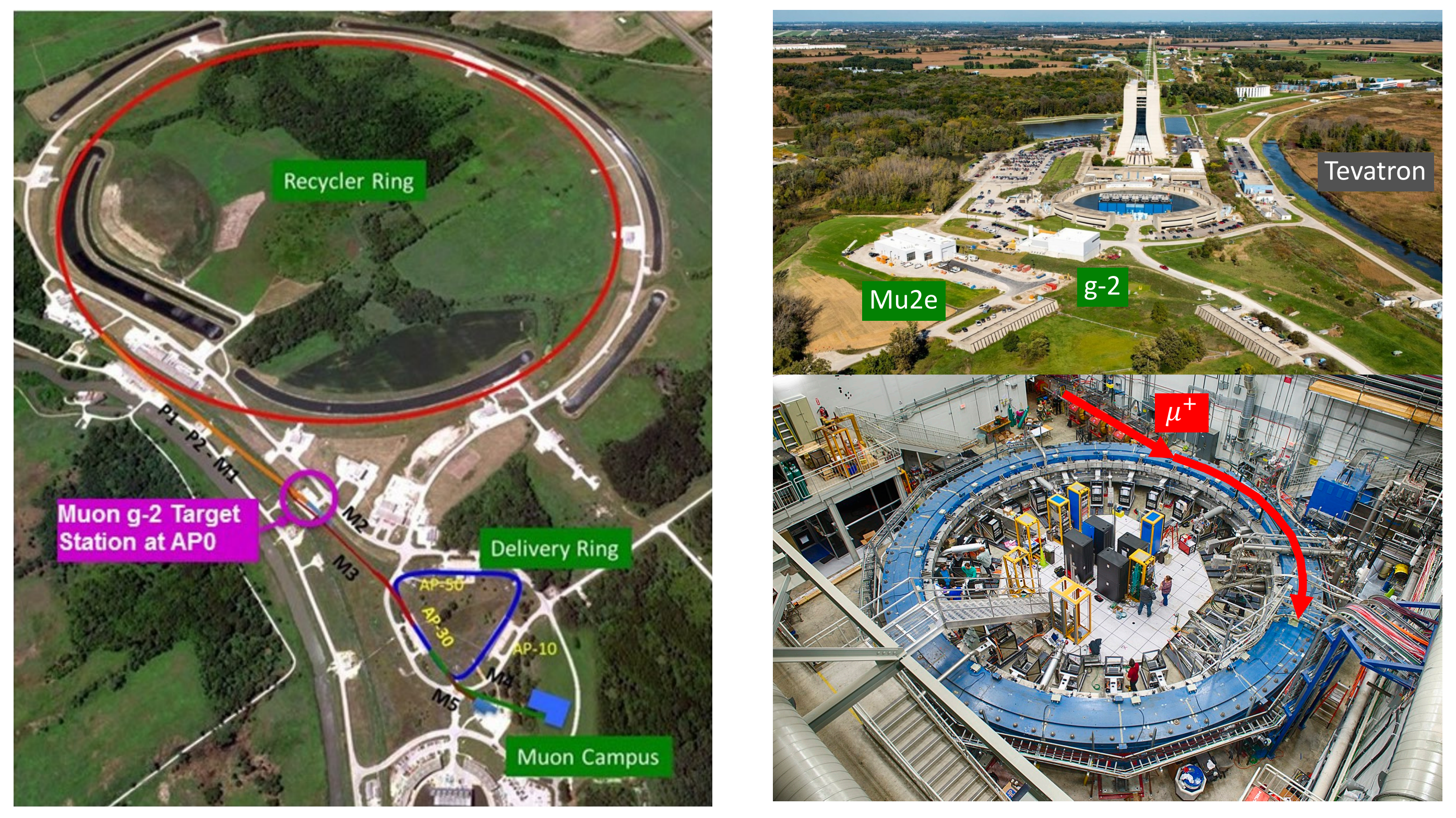
Magnetic moment $\quad \mu=g \frac{e}{2 m} s$
Classical: $\quad g=1$
$\begin{aligned} & \text { Dirac equation: } \quad g=2 \\ & i\left(\partial_{\mu}-i e A_{\mu}(x)\right) \gamma^{\mu} \psi(x)=m \psi(x)\end{aligned}$

Interactions w/ quantum foam: $\quad g>2 \quad a_{\mu}=\frac{g-2}{2}$ 


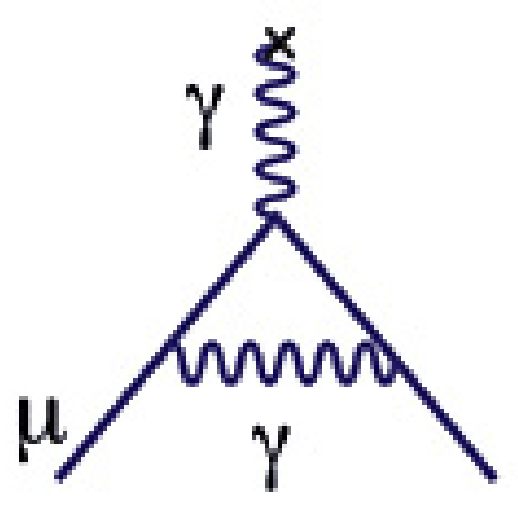

QED

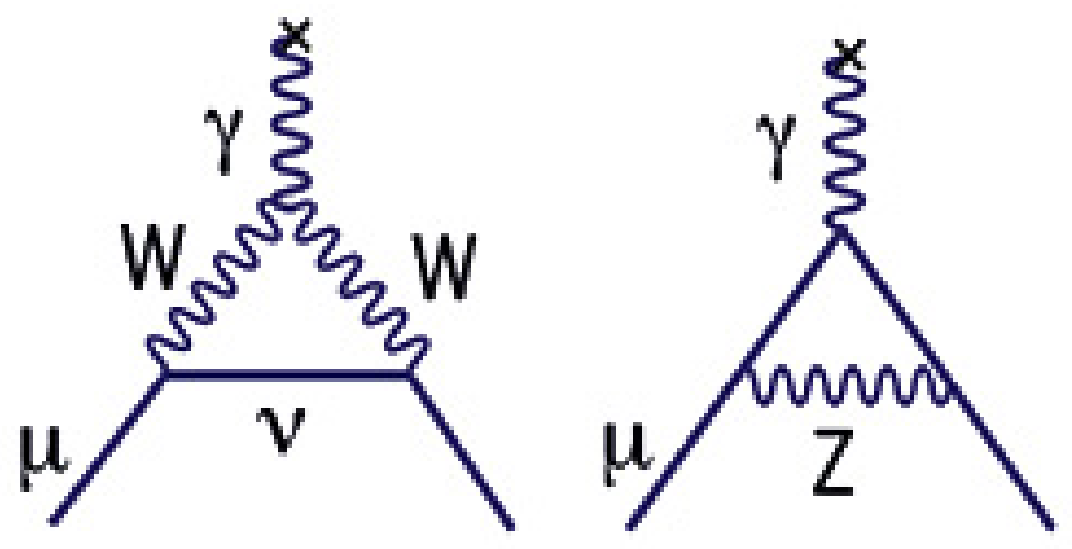

electroweak

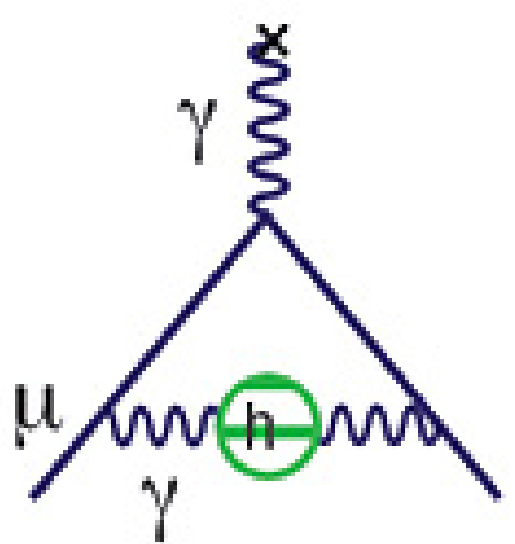

LO hadronic

Hadronic LO VP

$a_{\mu}=6894.6 \times 10^{-11}$

$\delta a_{\mu}=32.5 \times 10^{-11}$

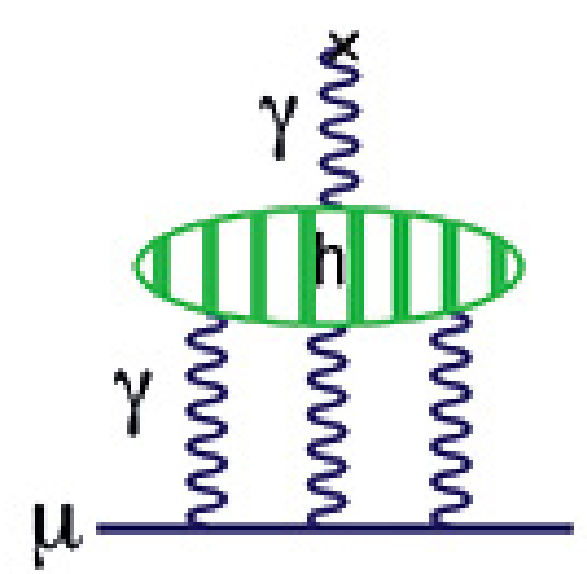

hadronic LbL

Hadronic LbL

$a_{\mu}=103.4 \times 10^{-11}$

$\delta a_{\mu}=28.8 \times 10^{-11}$
Theory:

Experiment (2021):
$(116591783 \pm 43) \times 10^{-11}$

$(116592061 \pm 41) \times 10^{-11}$ 


\section{Improvements in $a_{\mu}^{\text {Had, LO VP }}\left(\right.$ KNT18) Calculation of $a_{\mu}^{\text {Had, VP }}$ and $a_{\mu}^{\text {Had, LbL }}$ using Lattice QCD}

Direct energy scan: CMD-3, SND, KEDR

Radiative return: BABAR, KLOE/KLOE-2, BESIII

- From first principles

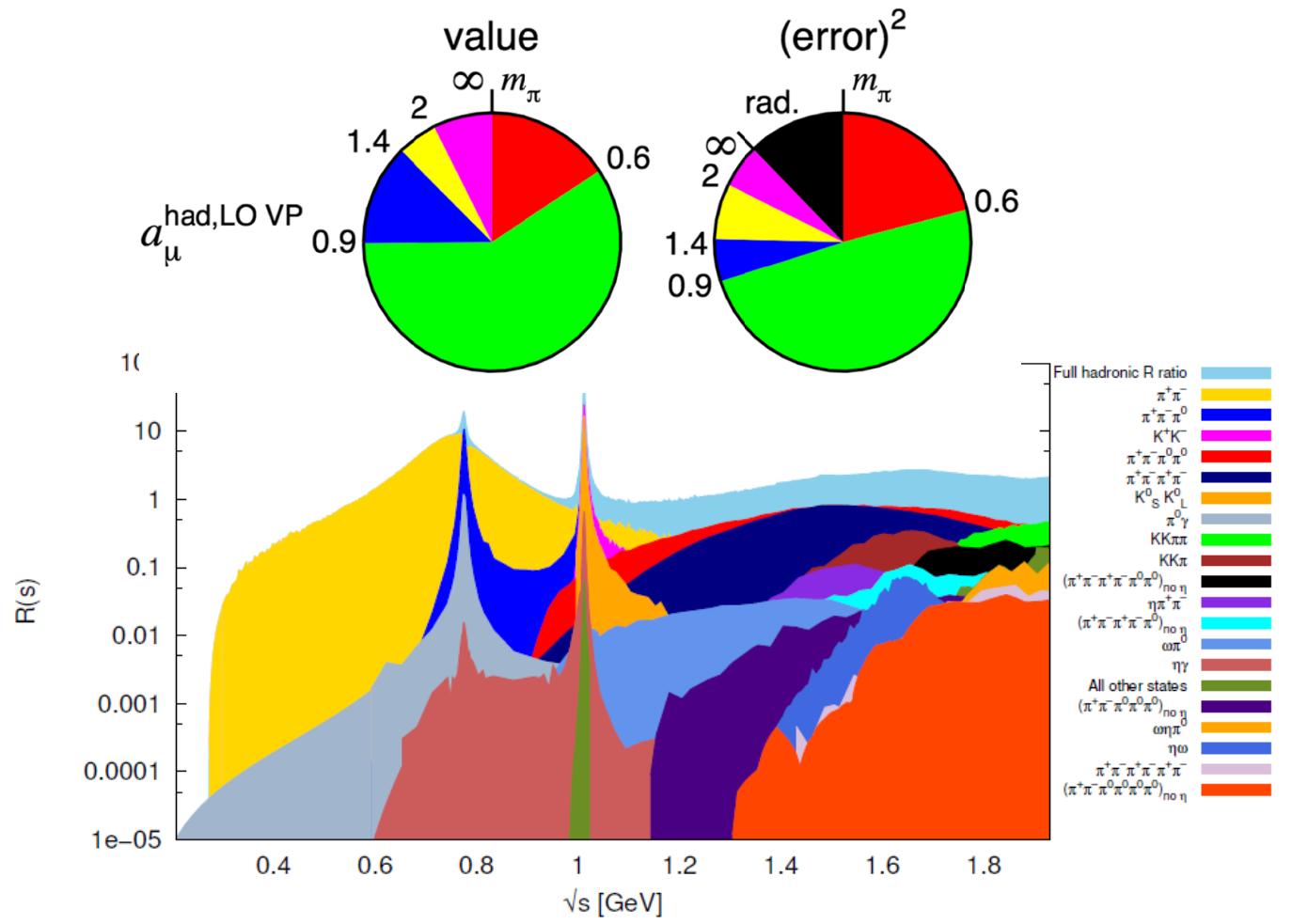

$a_{\mu}^{\mathrm{Had}, \mathrm{LO} \text { VP }}=(693.26 \pm 2.46) \times 10^{-10}$

- Can be used to improve R-ratio results

- Several collaborations working on this

- including RBC/UKQCD and Mainz

- Precision needs improvement; calculations ongoing

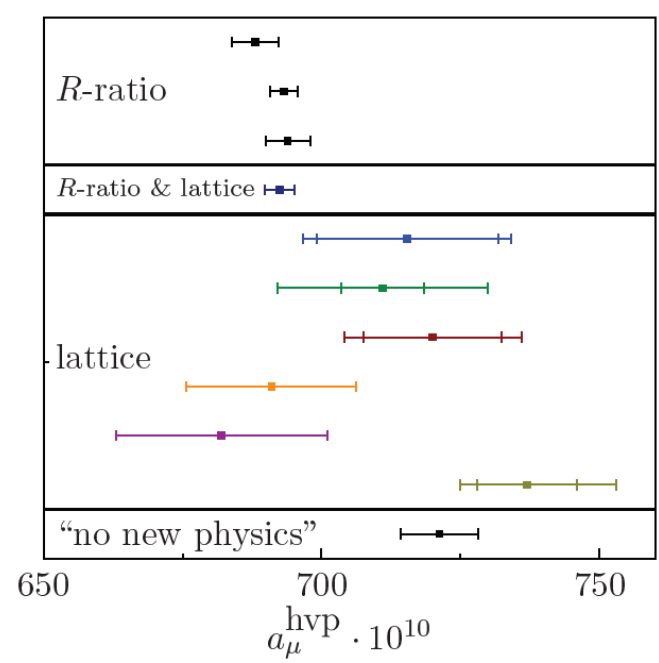

Jegerlehner 2017

Teubner et al 2018

Davier et al 2019

RBC/UKQCD 2018

RBC/UKQCD 2018

BMW 2017

CLS Mainz 2019

FermiLab/HPQCD/MILC 2019

ETMC 2019

PACS 2019

A. Keshavarzi, D. Nomura and T. Teubner, Phys. Rev. D 97, no. 11, 114025 (2018).

V. Gülpers, arXiv:2001.11898 [hep-lat] (2020). 


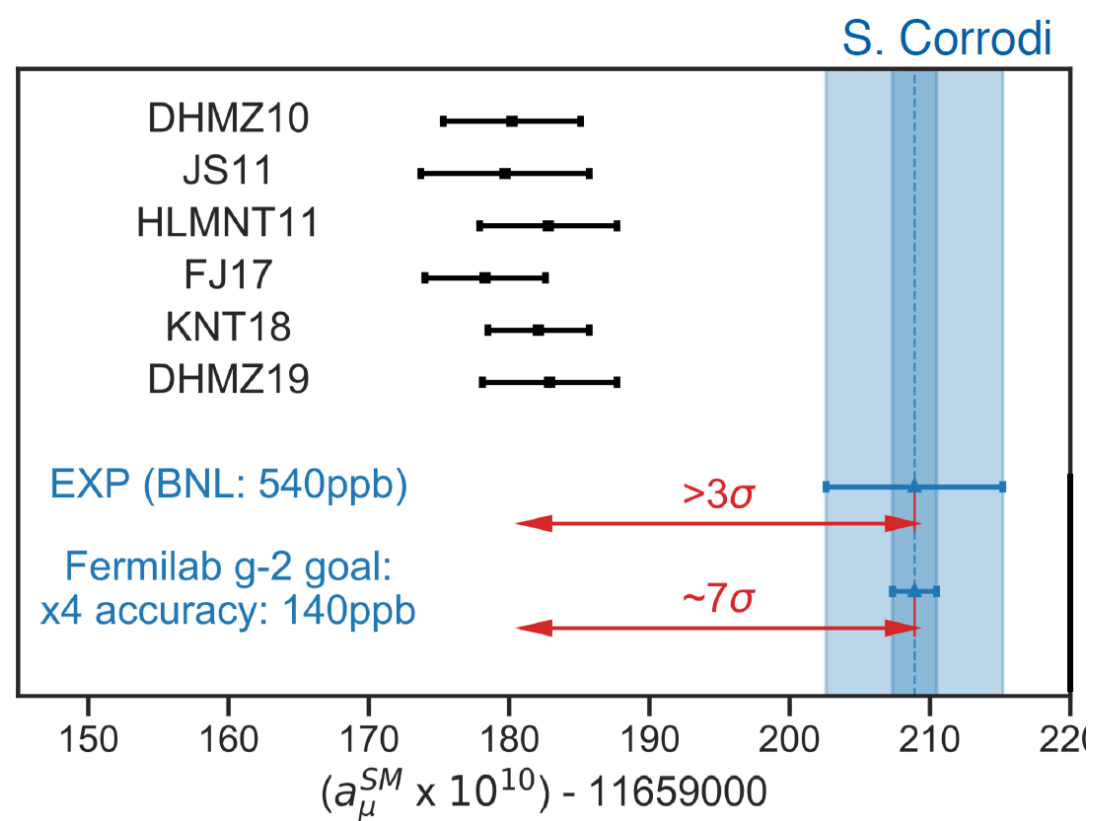

In case of a Beyond-SM $a_{\mu}$, some of the possible contributors to the respective discrepancy would be:

- Dark matter

- Supersymmetry (SUSY)

- Extra dimensions

- Additional Higgs Bosons [S. Iguro et al., arXiv:1907.09845 [hep-ph]]
Muon g-2 window in the search for inelastic dark matter (iDM) :
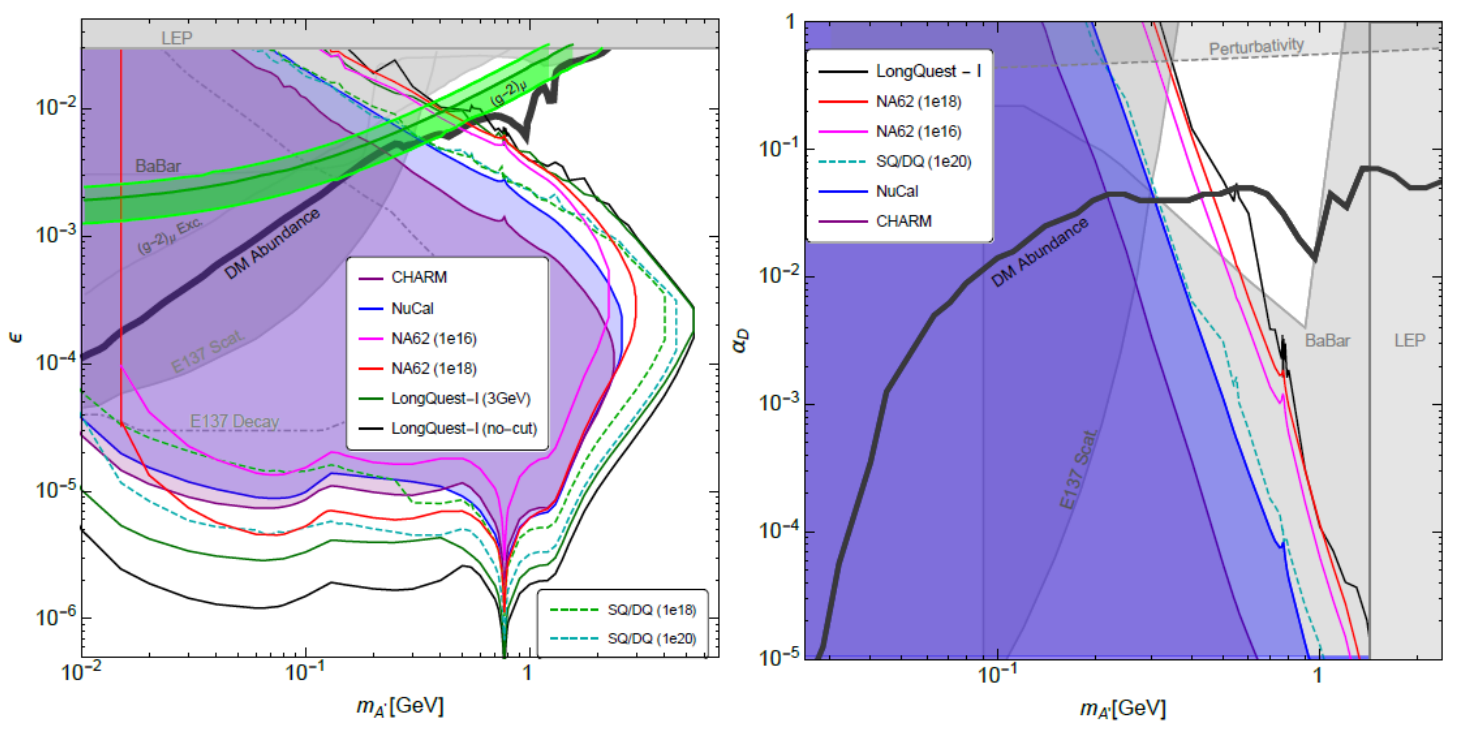
(b) iDM Muon $g-2$ Target: $\Delta=0.4, \epsilon=\epsilon_{(g-2)}$

$\left(a_{D}\right.$ : analogue of the fine structure constant for a new $U(1)$ gauge symmetry $U(1)_{D} . \Delta$ : mass splitting $\Delta=\frac{\left(m_{2}-m_{1}\right)}{m_{1}}$.)

NA62 Experiment at CERN is ongoing and may yield iDM results.

See Y.-D. Tsai et al., arXiv:1908.07525

[hep-ph] (2019). 


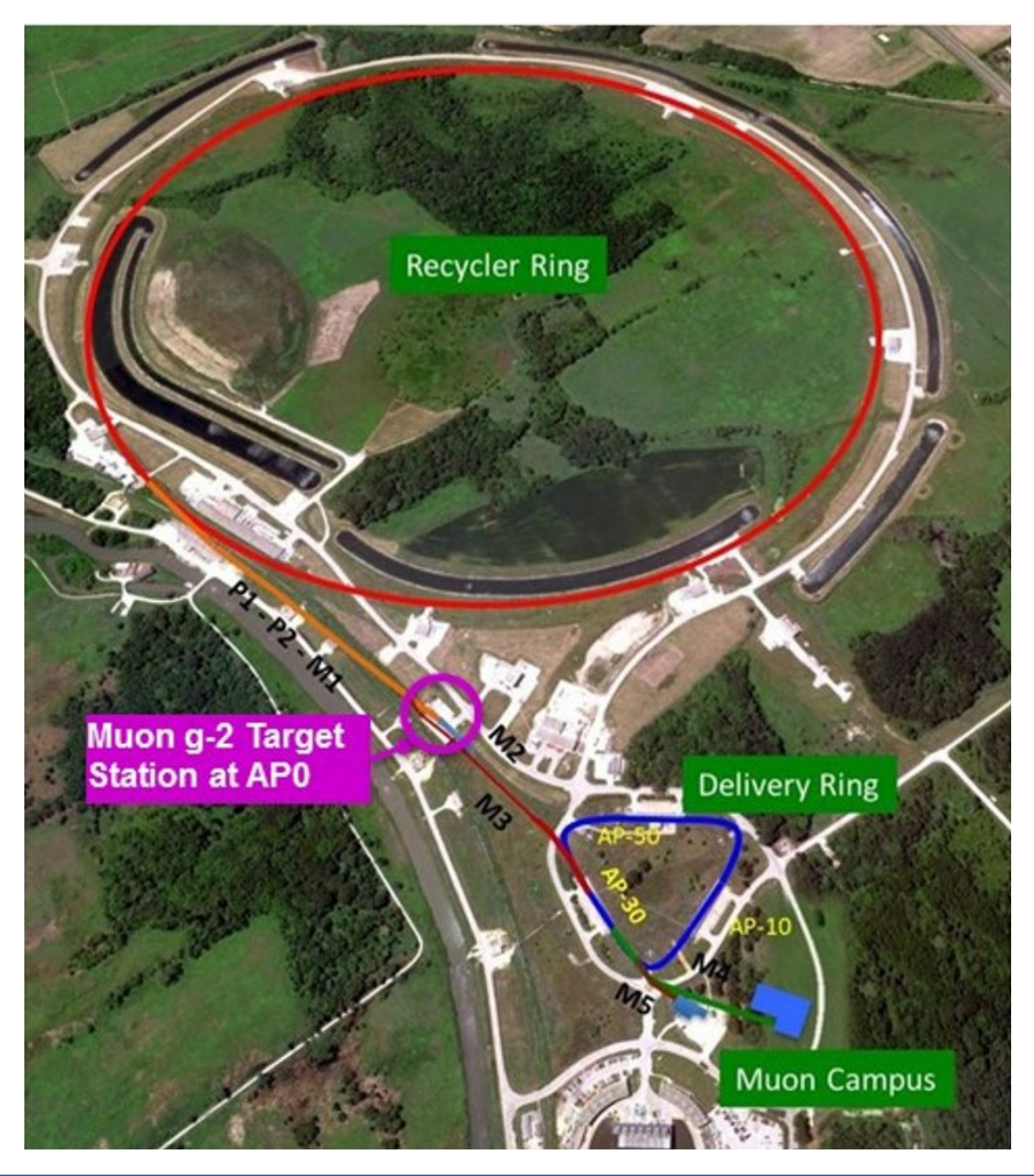

Improvements over the Muon $g$-2

Experiment at BNL (E821):

$>$ More muons, delivered more often to the storage ring

$>$ Improved muon storage function

$>$ Better beam dynamics modeling

$>$ Higher field uniformity and better field monitoring

$>$ Reduced spin precession frequency systematics 


\section{From BNL to FNAL: the Great Move}

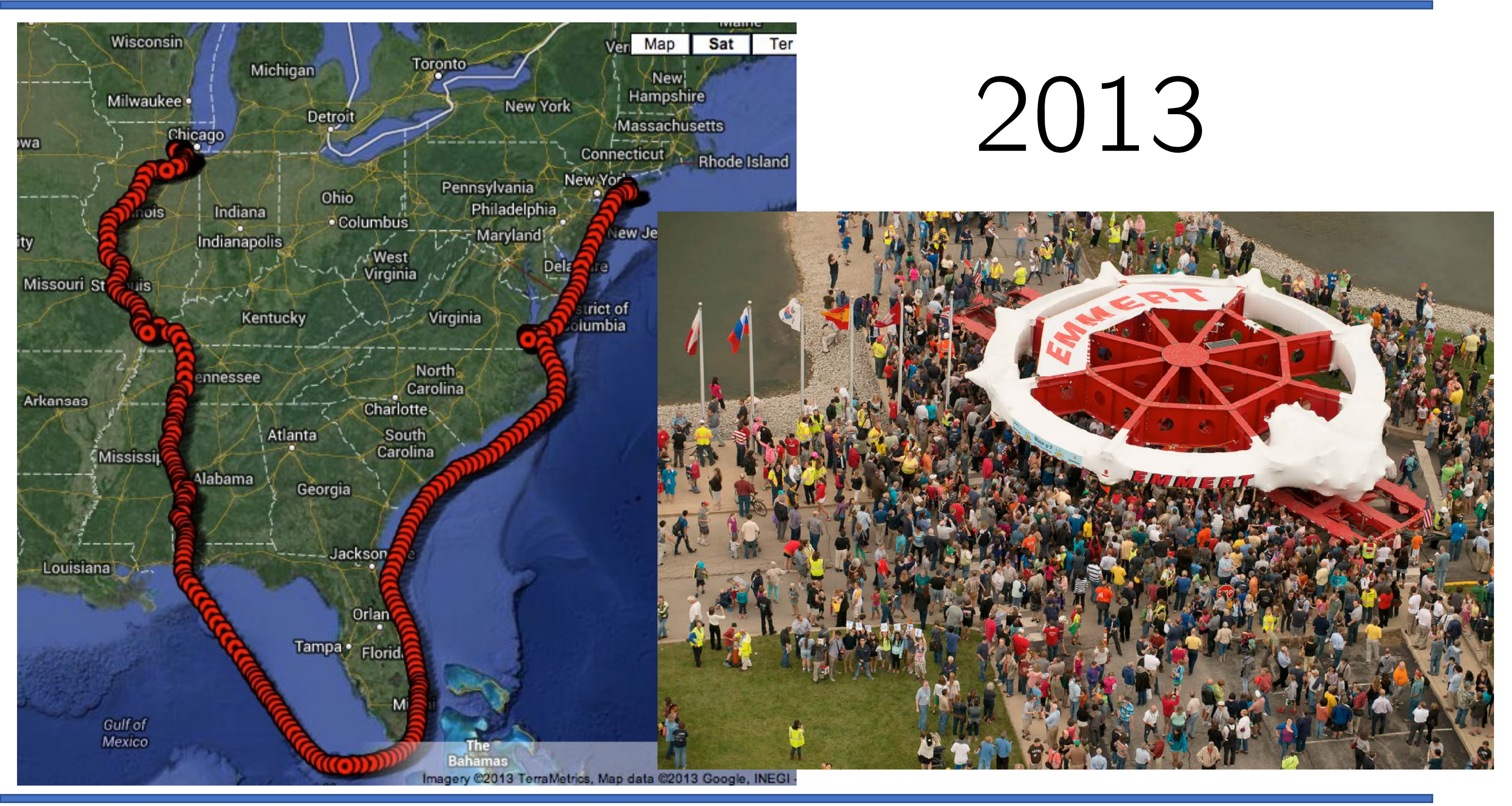




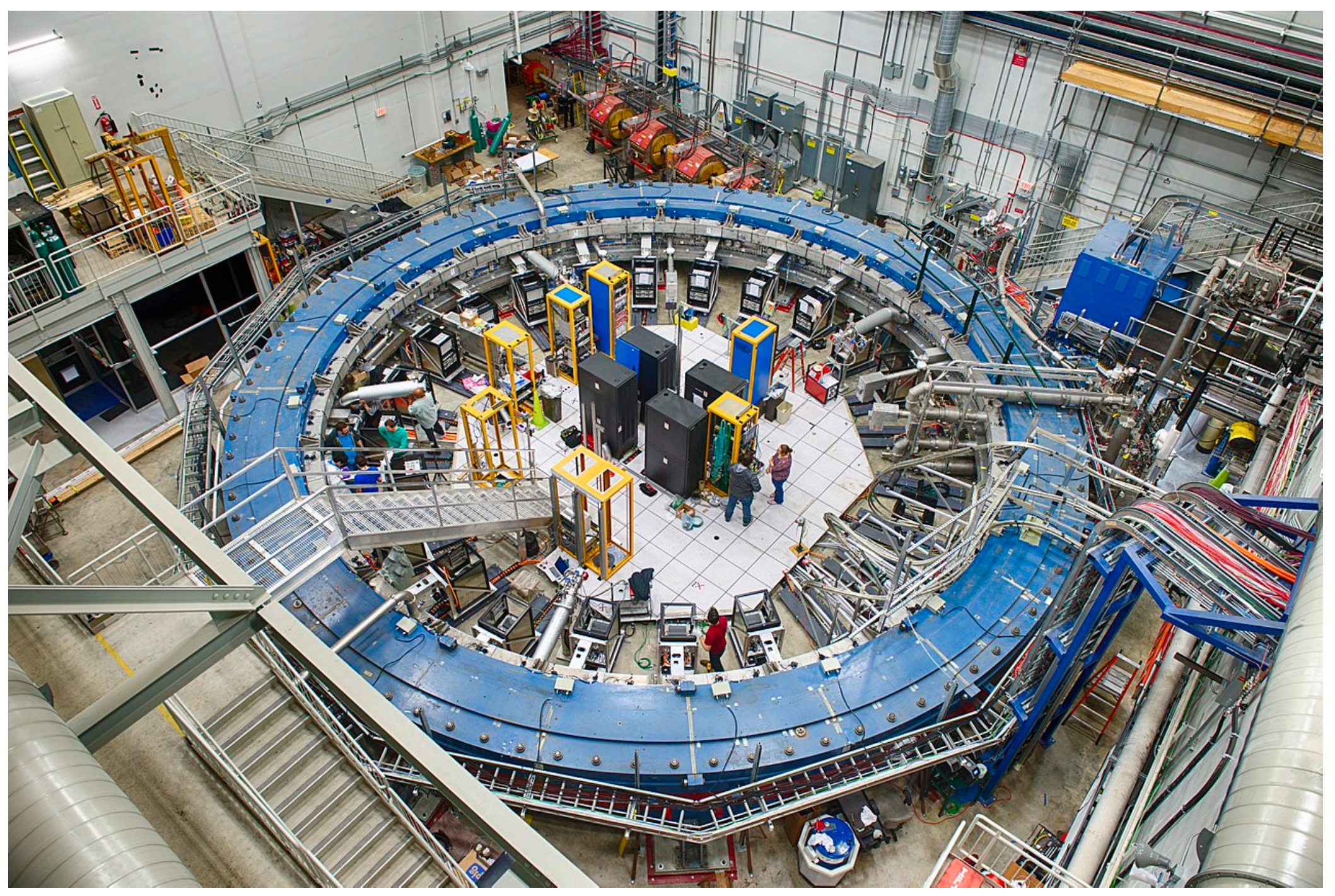

Technical design projection:

$>\sim 20 \mathrm{x}$ more data

$>\sim 3 \mathrm{x}$ reduction of systematic errors 


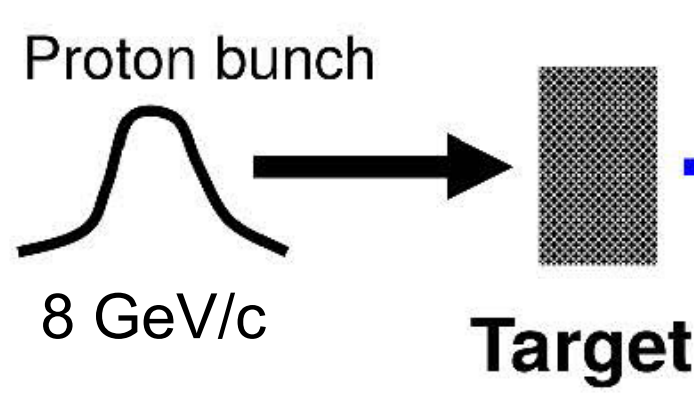

- Storage ring: $7 \mathrm{~m}$ radius toroidal C-magnet with $1.45 \mathrm{~T}$ magnetic field

- Inflector: cancels the $1.45 \mathrm{~T}$ main magnetic field for muons at injection

- Kickers deflect the injected muons onto the centerline orbit

- Electrostatic quadrupoles provide vertical beam focusing 


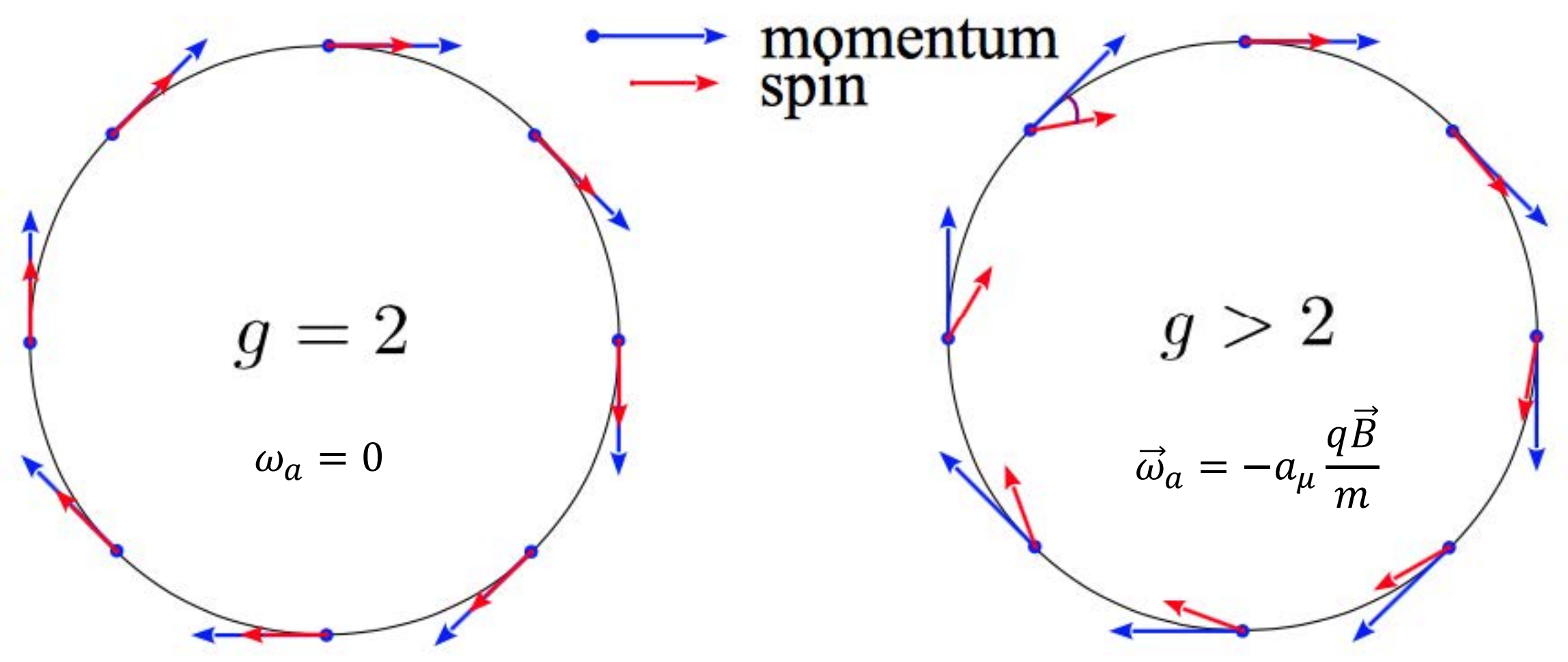

If $g=2$, the angle between the magnetic moment and the momentum does not change. If $g>2$, the angle between the magnetic moment and the momentum changes linearly. 

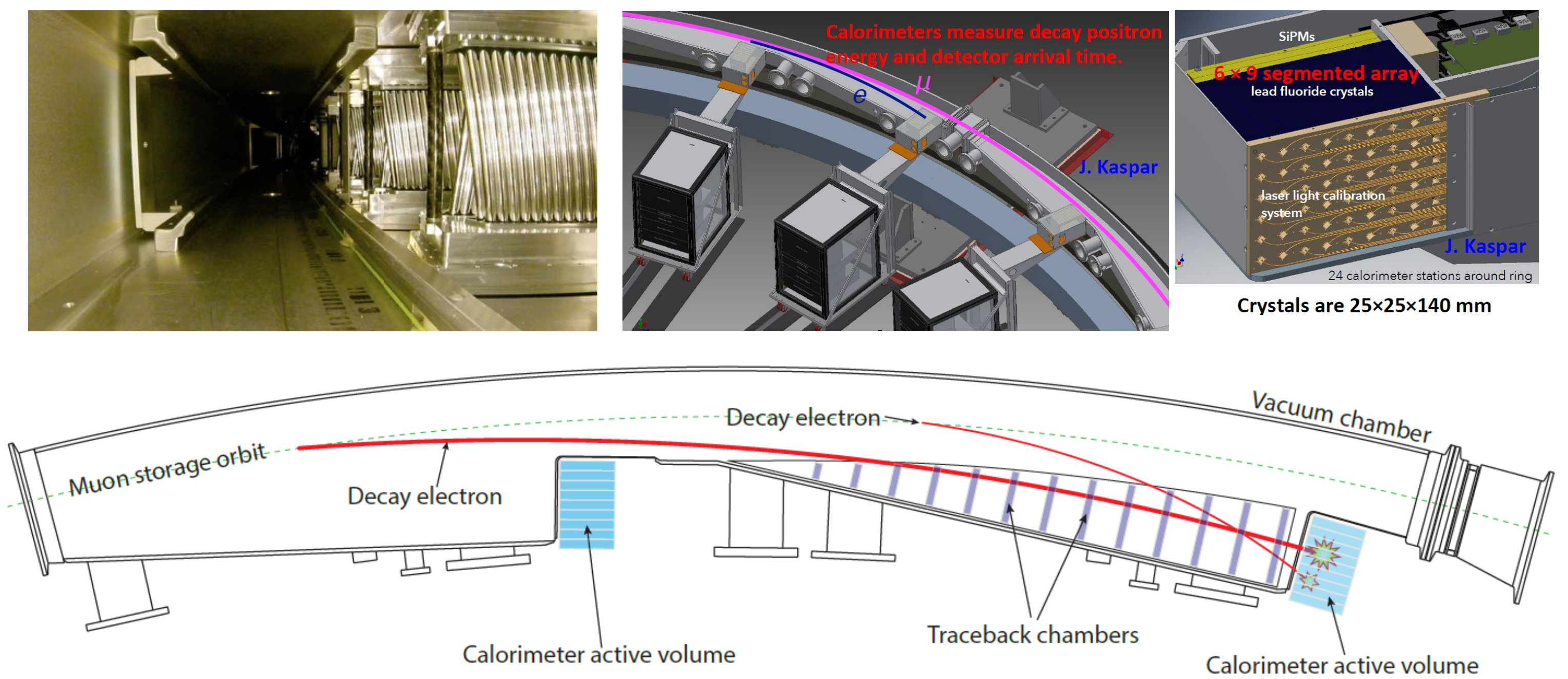

Straw trackers: reconstruct decay $e^{+}$trajectories Calorimeters: detect decay $e^{+}$energy and arrival times 


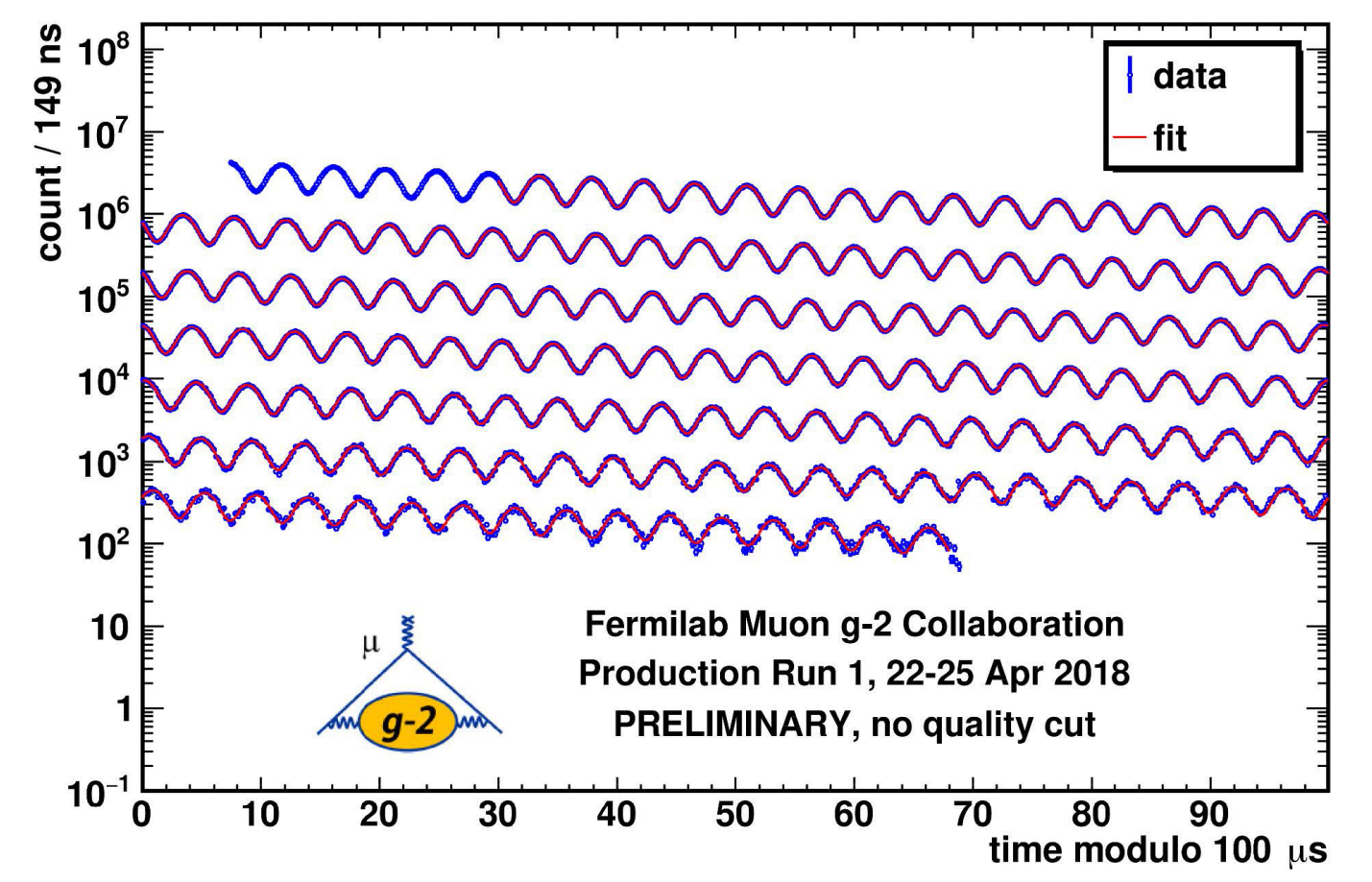

$f(t)=N_{0} e^{-\lambda t}\left[1+A \cos \left(\omega_{a} t+\phi\right)\right]$

$\lambda$ : exponential decay constant $\omega_{a}$ : muon anomalous precession frequency

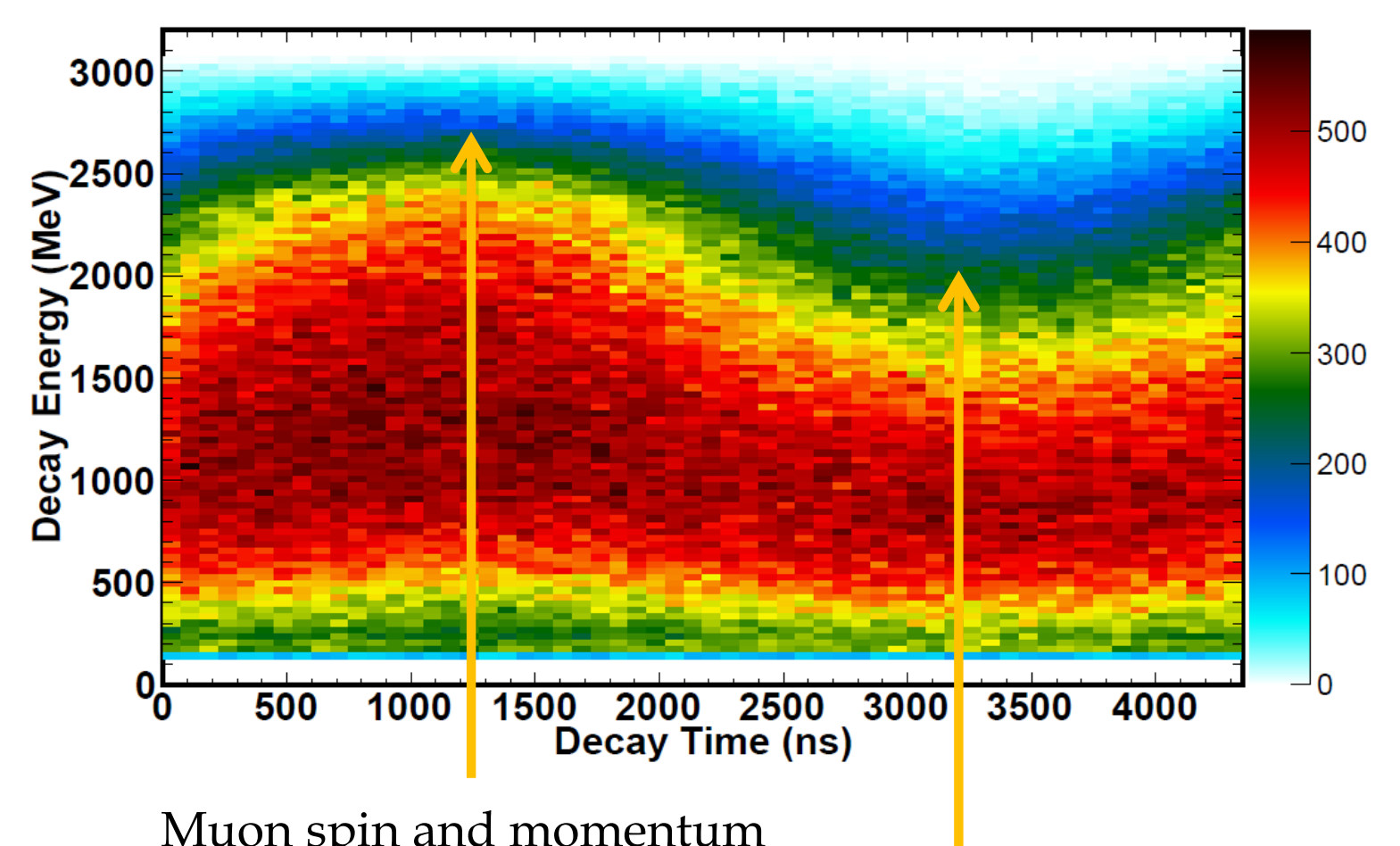

$$
\text { are aligned. Muon spin and momentum }
$$
are anti-aligned.

Early-to-late phase change:

If, $\phi=\phi(t)=\phi_{0}+\phi_{1} t$, then

$$
\cos \left(\omega_{a} t+\phi\right)=\cos \left(\omega_{a} t+\phi_{0}+\phi_{1} t\right)=
$$$$
=\cos \left(\left(\omega_{a}+\phi_{1}\right) t+\phi_{0}\right)
$$ 


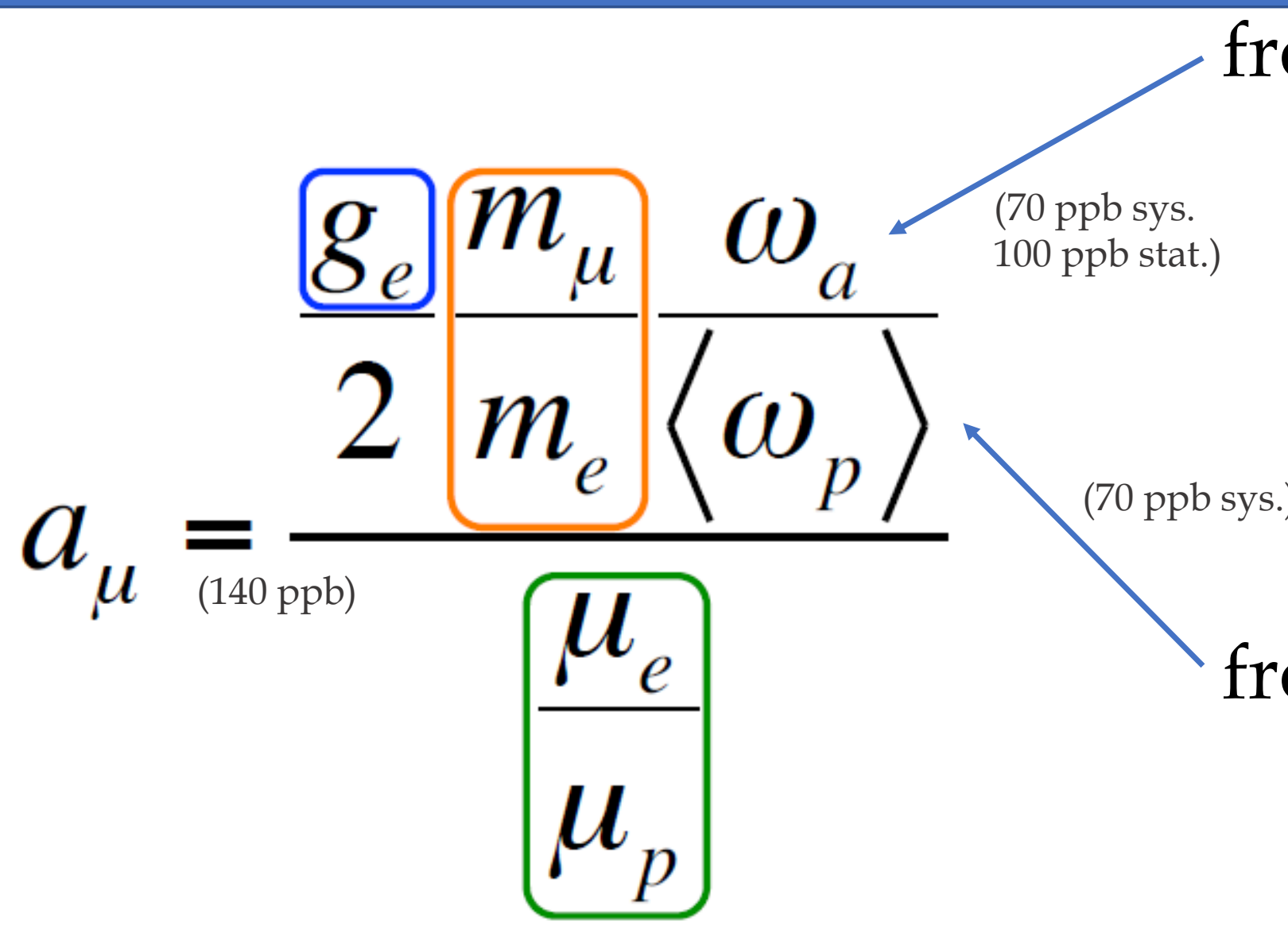

From CODATA [1]:

$\mathrm{g}_{\mathrm{e}}=-2.00231930436182(52)(0.00026 \mathrm{ppb})$

$\mathrm{m}_{\mu} / \mathrm{m}_{\mathrm{e}}=206.7682826(46)(22 \mathrm{ppb})$

$\mu_{\mathrm{e}} / \mu_{\mathrm{p}}=-658.2106866(20)(3.0 \mathrm{ppb})$ 


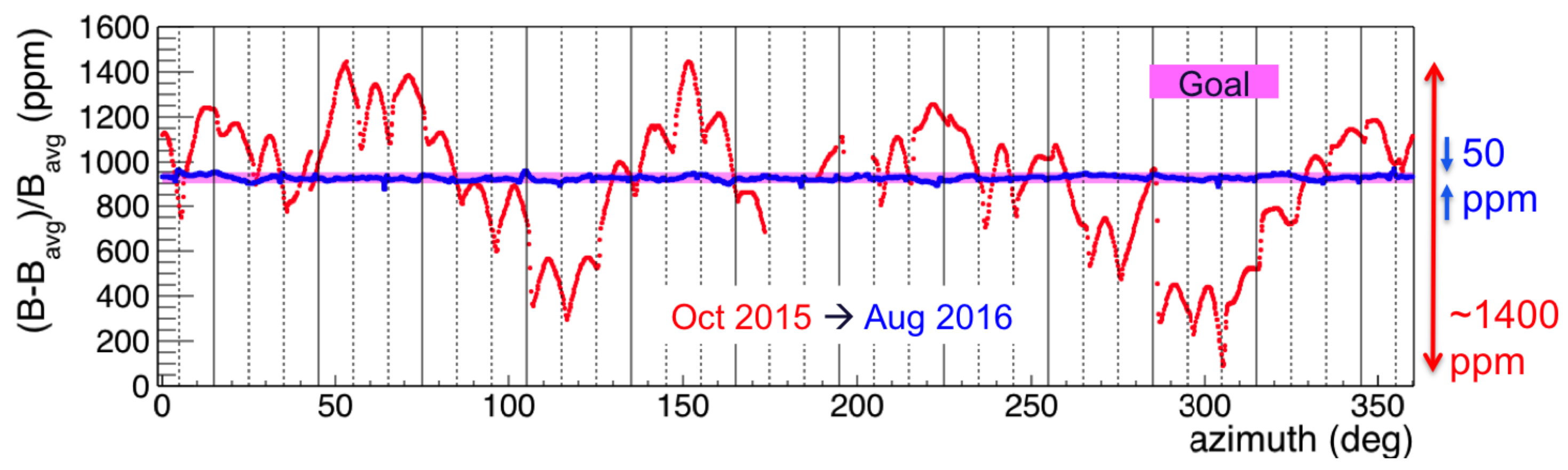

$>$ Passive shimming is performed by inserting tiny metal pieces to increase the field.

$>$ Magnetic field was made $3 \times$ more uniform than at BNL.

$>$ Active shimming is also used. 
Fixed probes on vacuum chambers

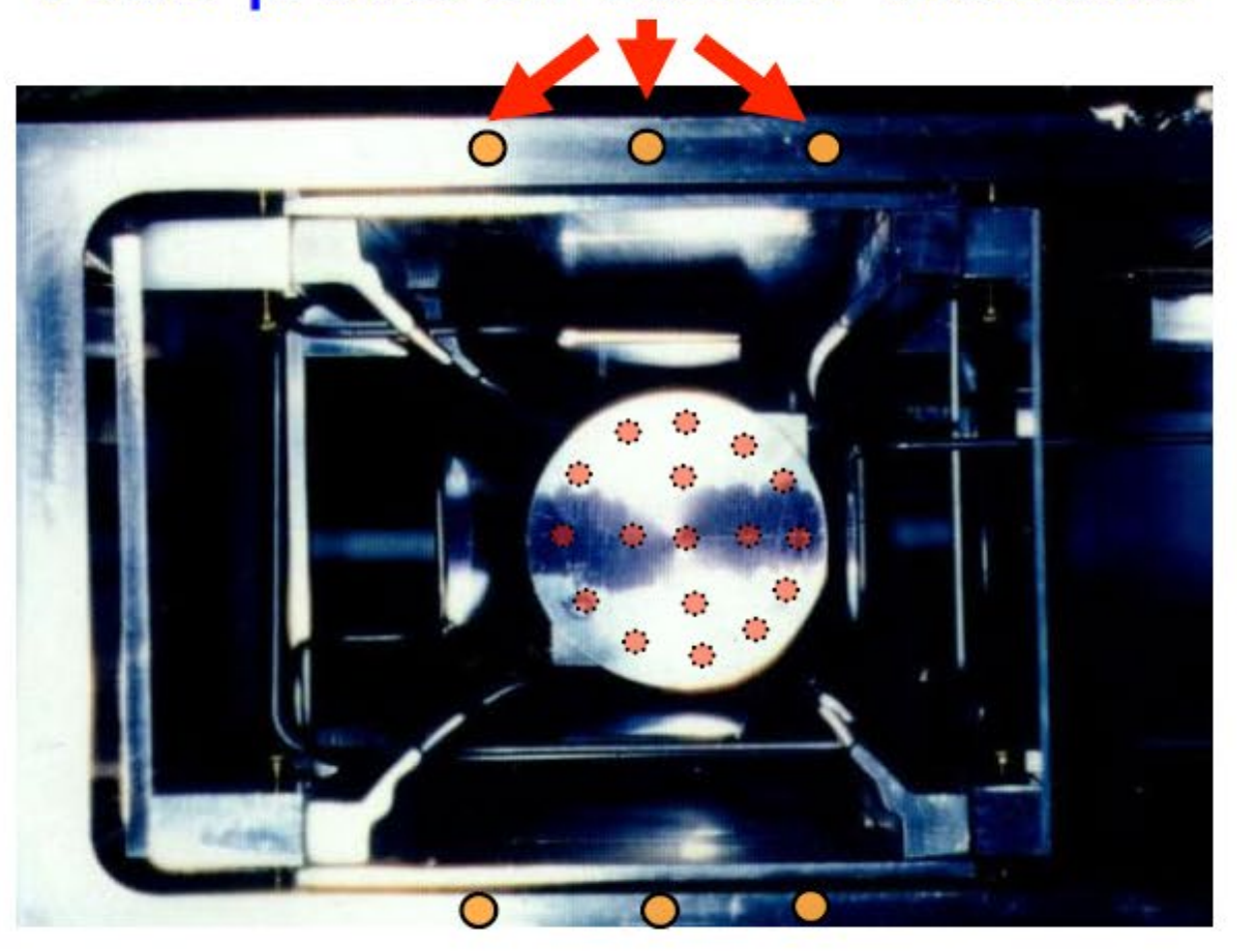

Trolley with matrix of 17 NMR probes

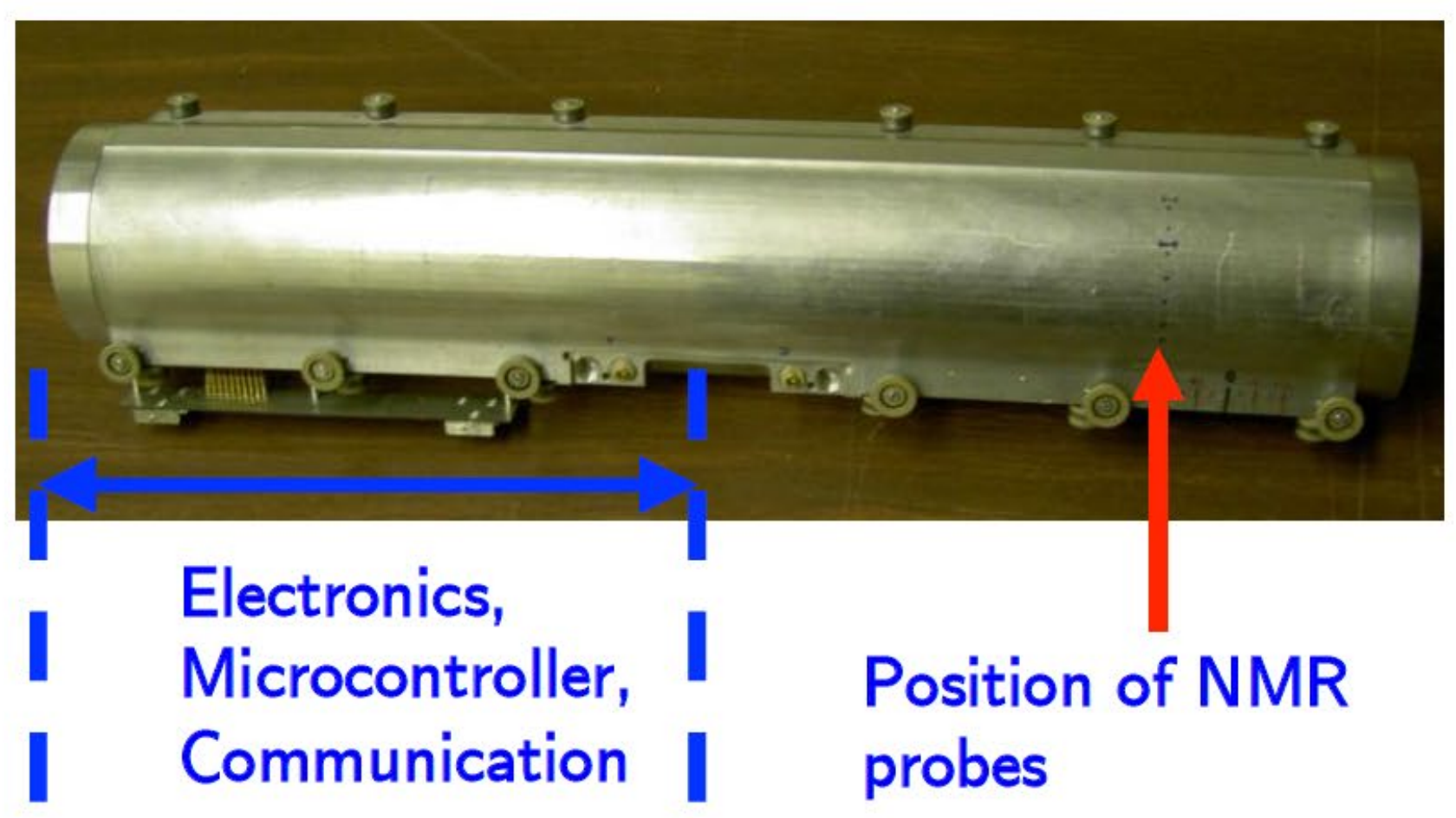




\begin{tabular}{|c|c|c|c|}
\hline & Correction & Uncertainty & Design goal \\
\hline$\omega_{a}^{m}$ (statistical) & - & 434 & 100 \\
\hline$\omega_{a}^{m}$ (systematic) & - & 56 & \\
\hline base clock & - & 2 & \\
\hline$C_{e}$ & 489 & 53 & \\
\hline$C_{p}$ & 180 & 13 & \\
\hline$C_{m l}^{r}$ & -11 & 5 & \\
\hline$C_{p a}$ & -158 & 75 & \\
\hline$\omega_{a}$ beam dynamics corrections $\left(C_{e}+C_{p}+C_{m l}+C_{p a}\right)$ & 499 & 93 & \\
\hline$\omega_{a}$ total systematic & 499 & 109 & 70 \\
\hline$\omega_{p}^{\prime}(T)(x, y, \varphi)$ & - & 54 & \\
\hline$M(x, y, \varphi)$ & - & 17 & \\
\hline$\left\langle\omega_{p}^{\prime}(T)(x, y, \varphi) \times M(x, y, \varphi)\right\rangle$ & - & 56 & \\
\hline$B_{q}$ & -17 & 92 & \\
\hline$B_{k}$ & -27 & 37 & \\
\hline$\tilde{\omega}_{p}^{\prime}(T)$ transient fields corrections $\left(B_{q}+B_{k}\right)$ & -44 & 99 & \\
\hline$\tilde{\omega}_{p}^{\prime}(T)$ total & 44 & 114 & 70 \\
\hline$\omega_{a} / \tilde{\omega}_{p}^{\prime}(T)$ total systematic & 544 & 157 & 100 \\
\hline external measurements & - & 25 & \\
\hline total [correction is for $\omega_{a} / \tilde{\omega}_{p}^{\prime}(T)$ ] & 544 & 462 & 140 \\
\hline
\end{tabular}


In the following eight or nine slides, I will talk about some of my recent personal contributions:

$>$ end-to-end simulations

$>$ application of simulation results to muon loss systematics 


\section{Beamlines of the Muon Campus}

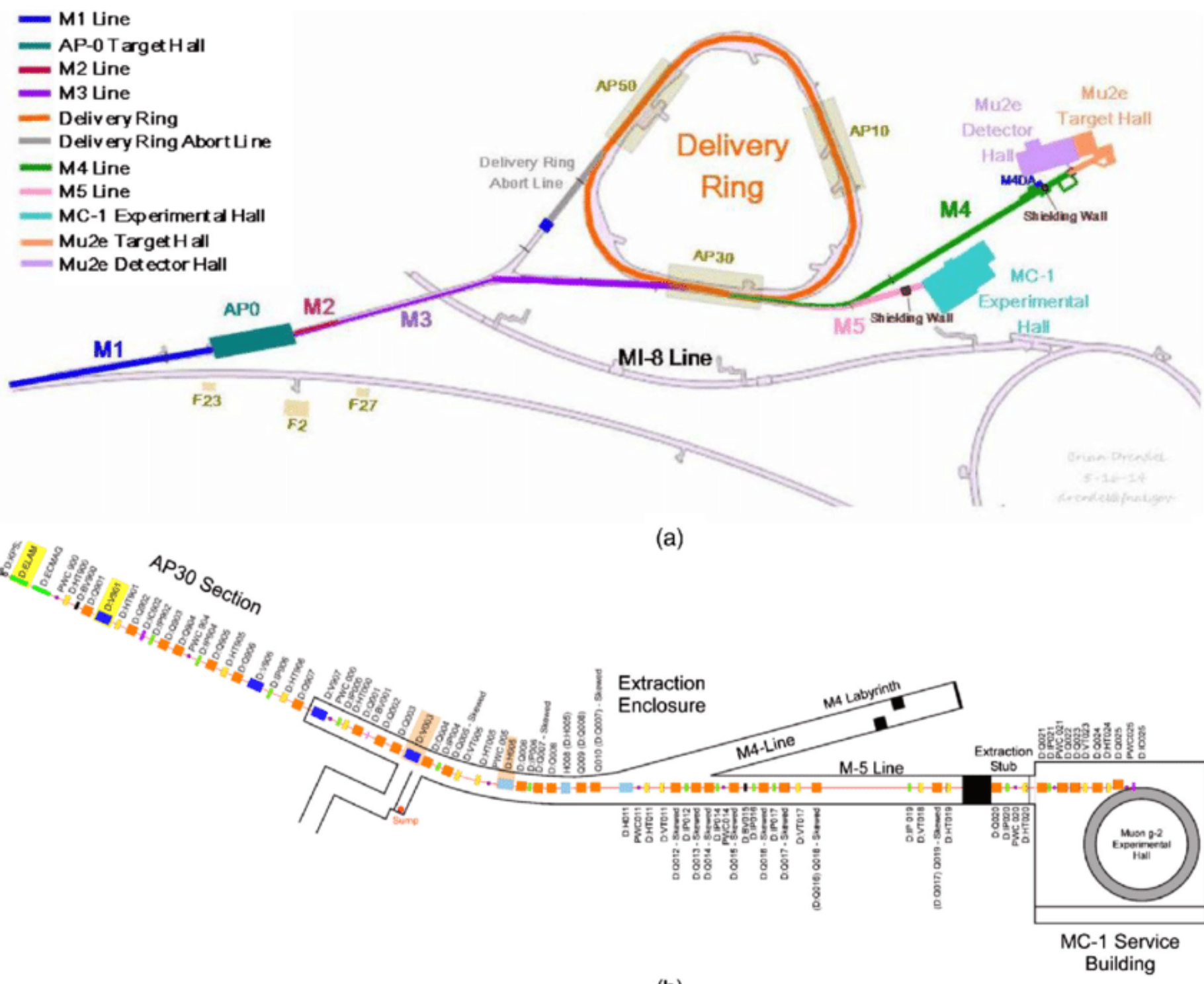

(b) 


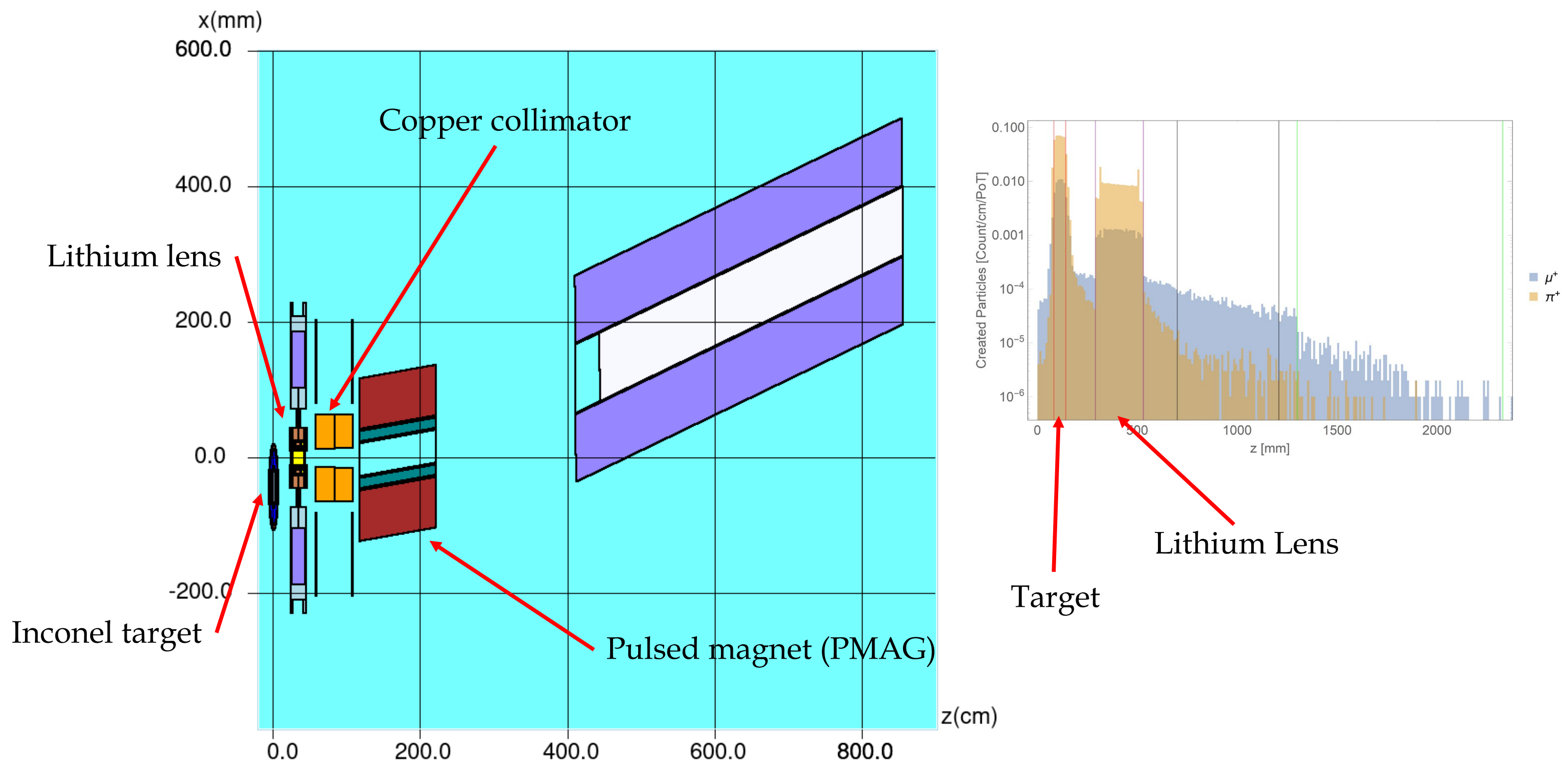




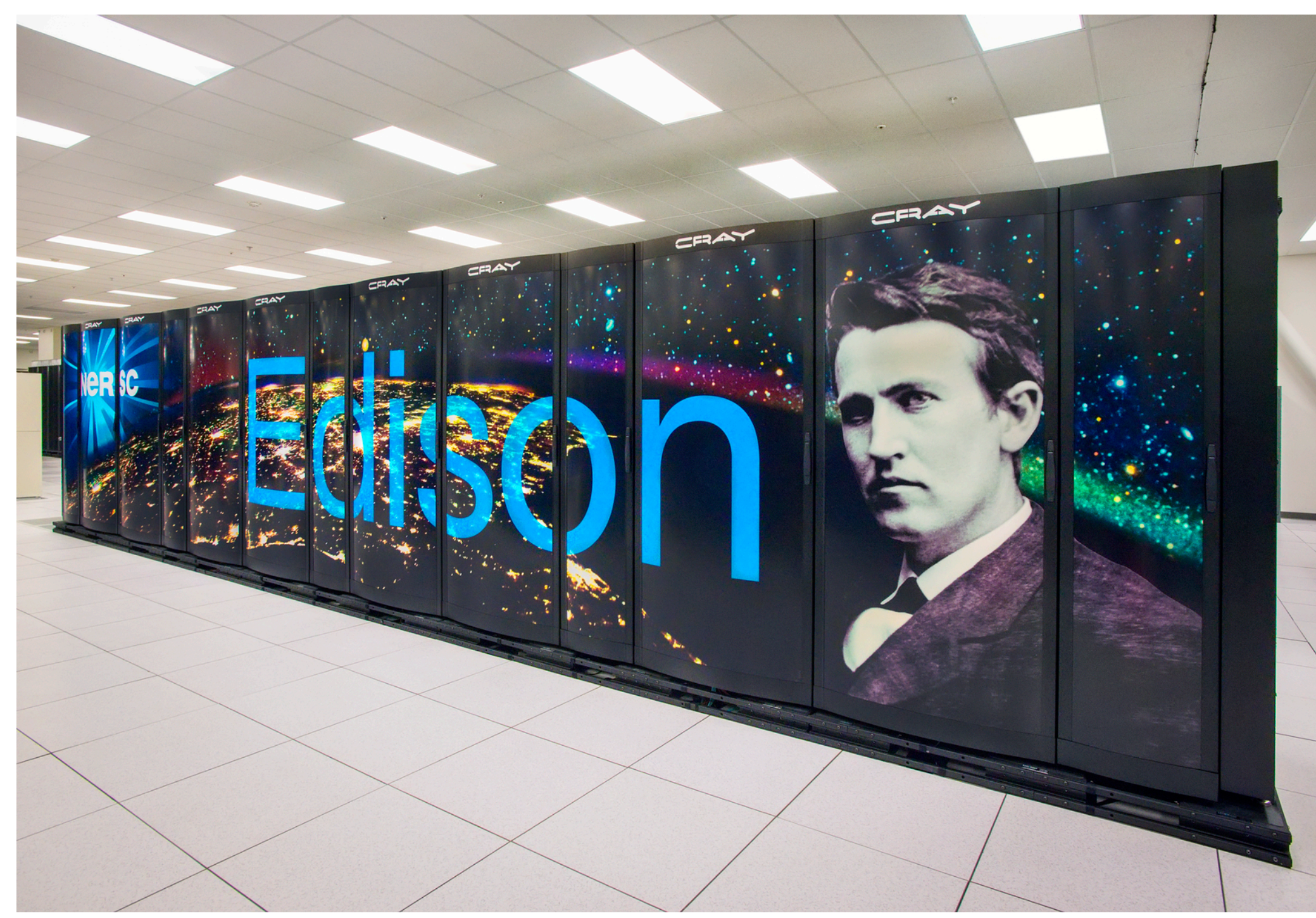

$2 \times 10^{13}$ protons

on target

HPC systems:

- NERSC

- $\quad$ Edison (2013-2019):

2.57 PFLOPS

- $\quad$ Cori (2015-): 30 PFLOPS

- $\quad$ Open Science Grid

- Up to 10000 cores at a time

- FermiGrid

Simulation tools:

- gm2ringsim (Geant4)

- COSY INFINITY

- BMAD

- MARS

- G4Beamline (Geant4) 
Simulation results, preliminary
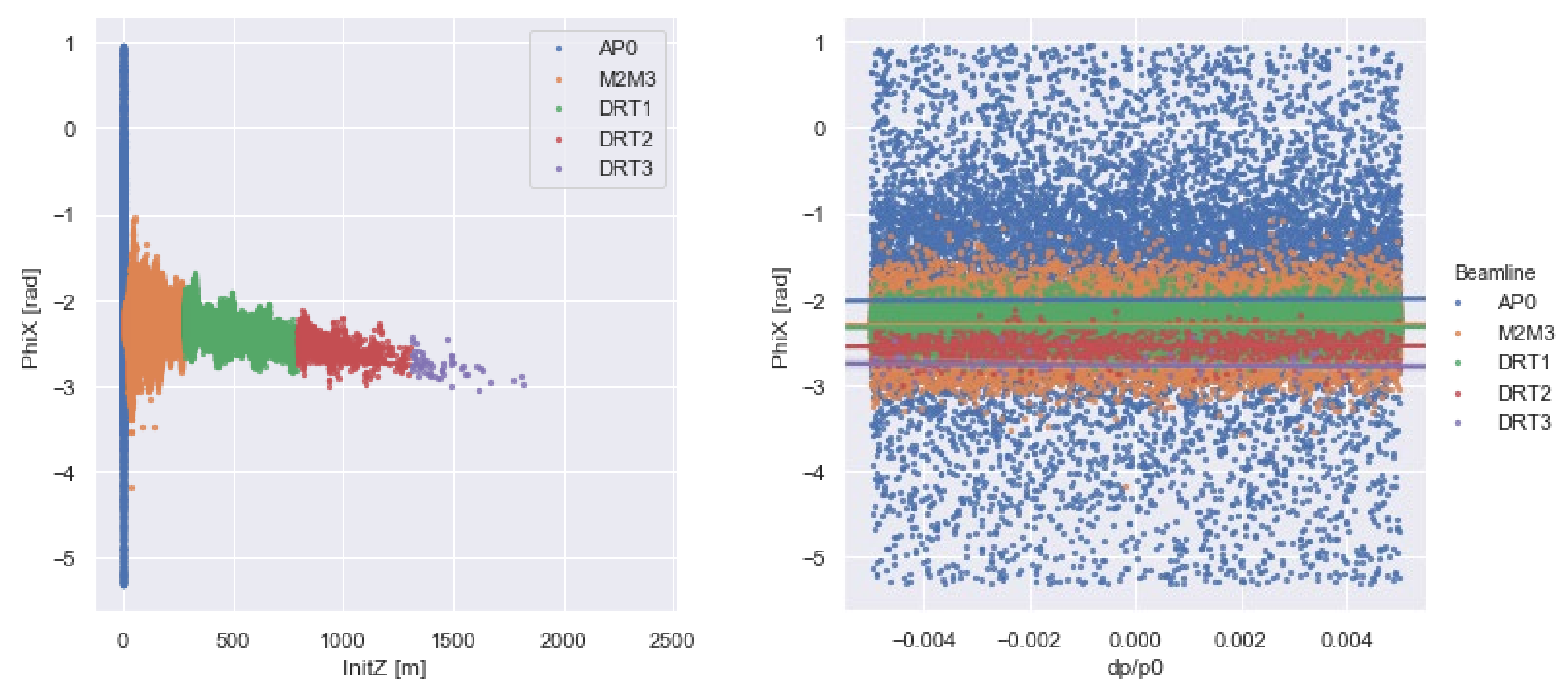

InitZ: muon creation location. PhiX: muon spin phase at entrance into the ring. $\mathrm{dp} / \mathrm{p} 0$ : momentum deviation. All data within $\left|\frac{d p}{d p 0}\right|<0.5 \%$, i.e. $3 \sigma$ acceptance of the storage ring. 
Experimental data: Hannah Binney.

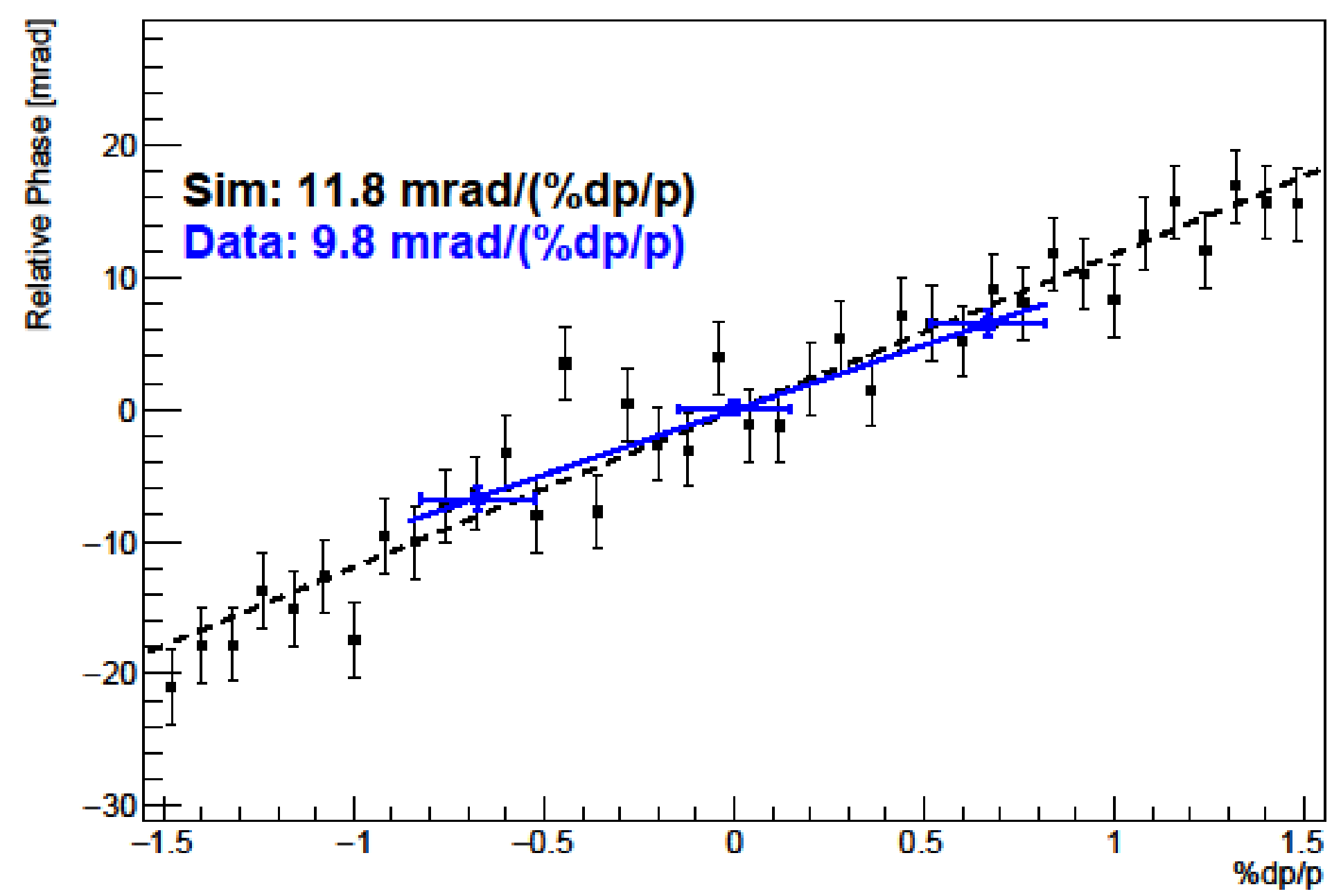

A real momentum dependence of the initial phase develops because of magnetic dipoles in the Delivery Ring.

Experimental data: based on runs with muon storage with higher or lower momenta. 
Simulation results (Mike Syphers)
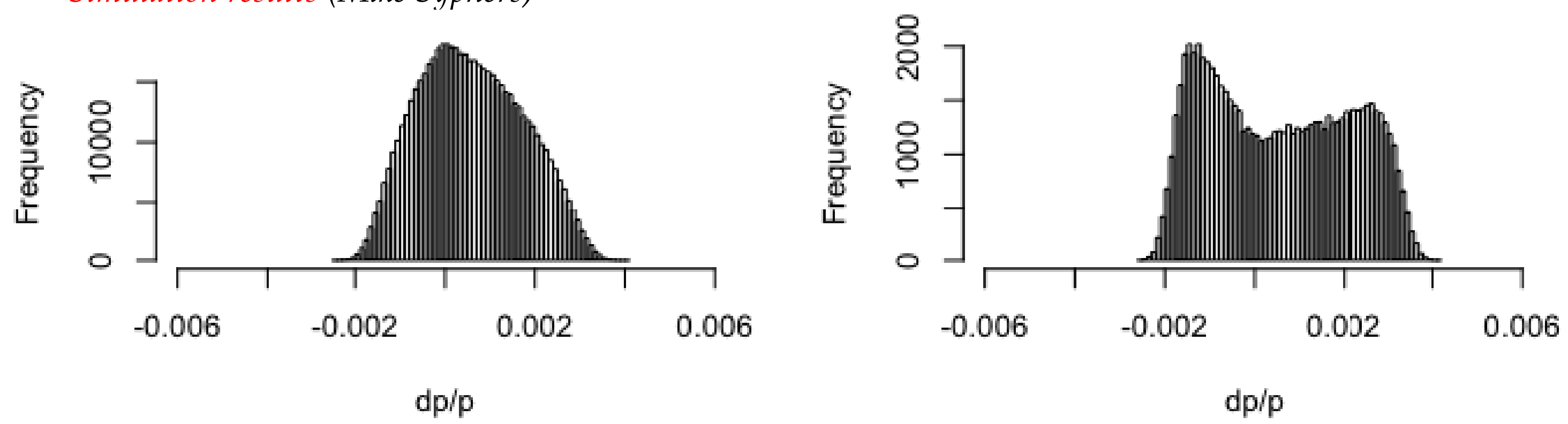

A -11(5) ppb correction due to muon losses.

Far below the overall $70 \mathrm{ppb}$ systematic error on the spin precession. Meeting the TDR goal of $20 \mathrm{ppb}$. 
The Decision to Unblind: a Remote Meeting of the Collaboration
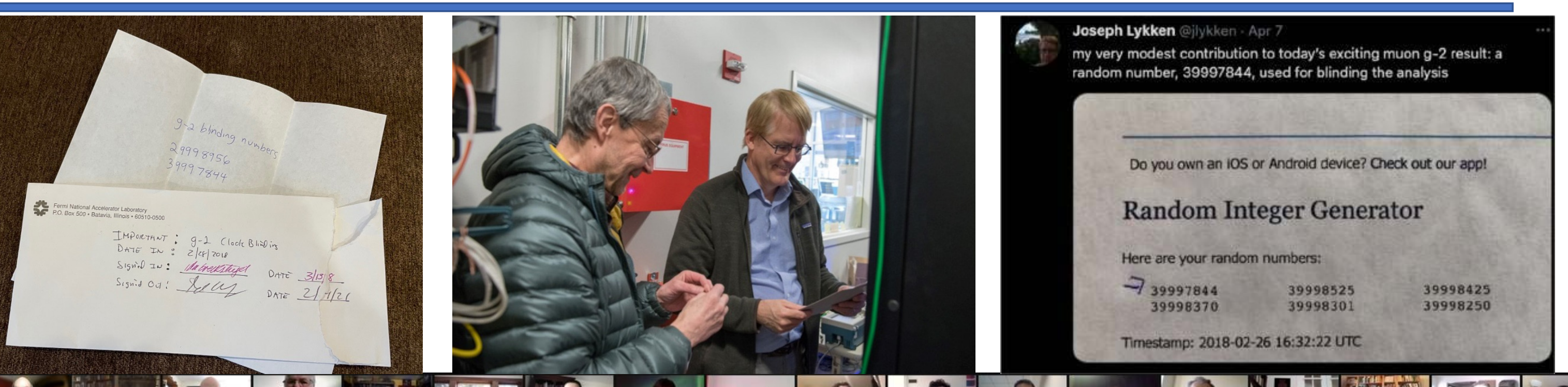

24

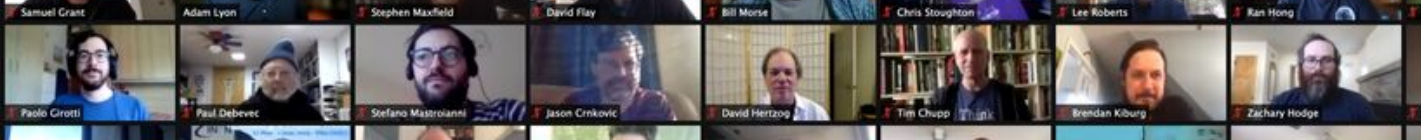

a [R L

政

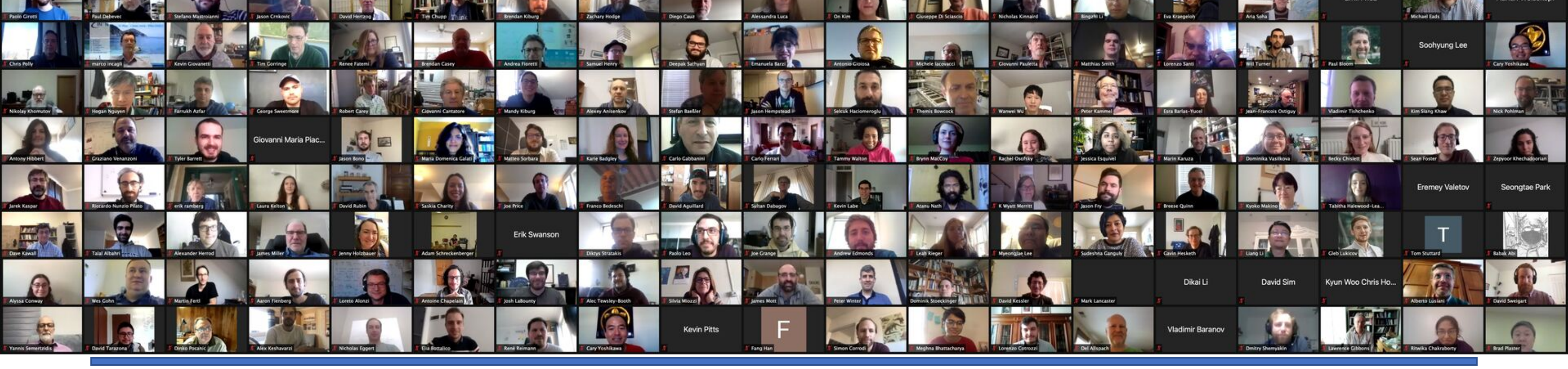




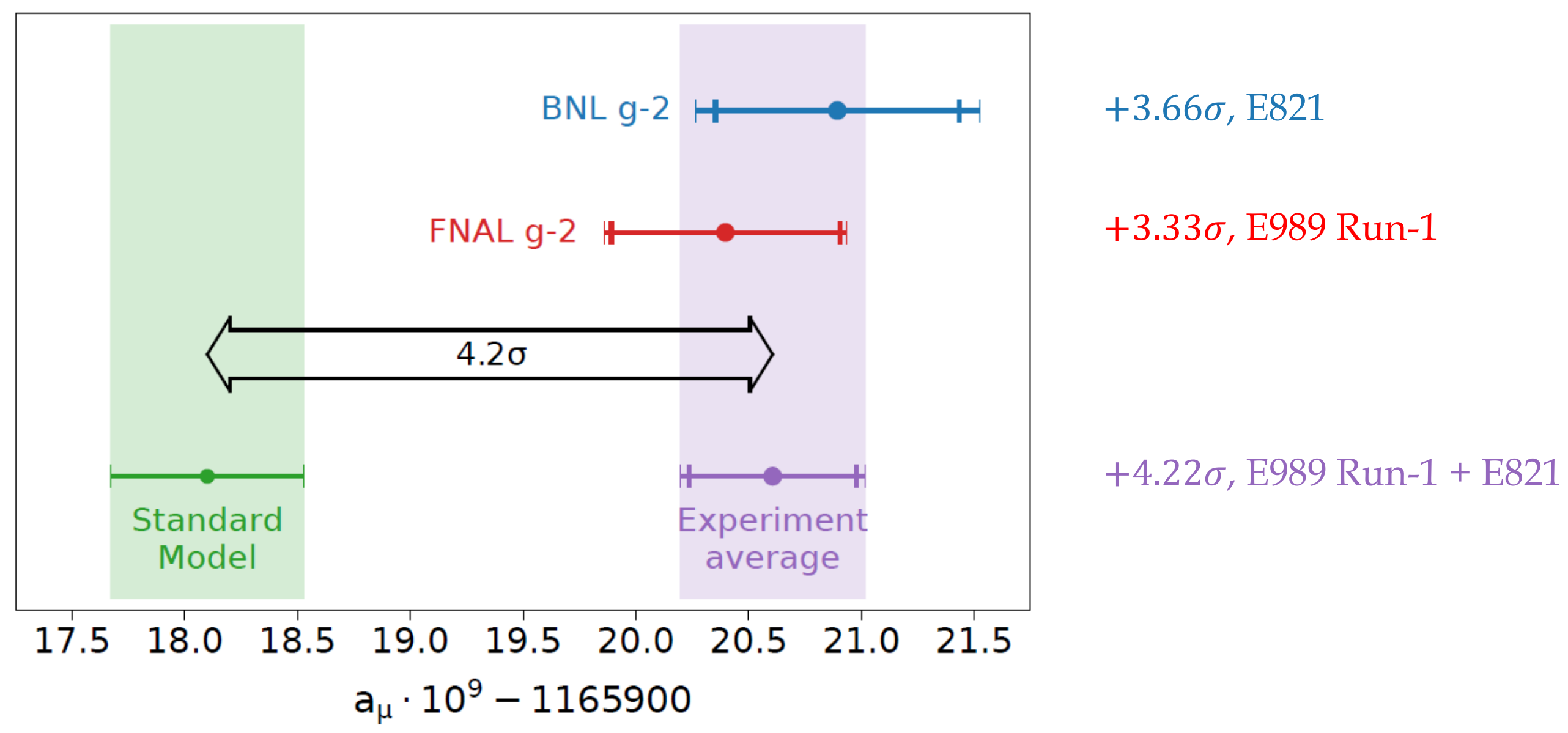

The SM value is the Muon g-2 Theory Initiative recommended value.

T. Aoyama et al., Phys. Rep. 887, 1 (2020). 


\section{Run-1 papers}

$>$ Measurement of the Positive Muon Anomalous Magnetic Moment to $0.46 \mathrm{ppm}$

https://doi.org/10.1103/PhysRevLett.126.141801

$>$ Measurement of the anomalous precession frequency of the muon in the Fermilab Muon $g$-2 Experiment https://doi.org/10.1103/PhysRevD.103.072002

$>$ Magnetic-Field Measurement and Analysis for the Muon g-2 Experiment at Fermilab

https://doi.org/10.1103/PhysRevA.103.042208

$>$ Beam dynamics corrections to the Run-1 measurement of the muon anomalous magnetic moment at Fermilab https://doi.org/10.1103/PhysRevAccelBeams.24.044002

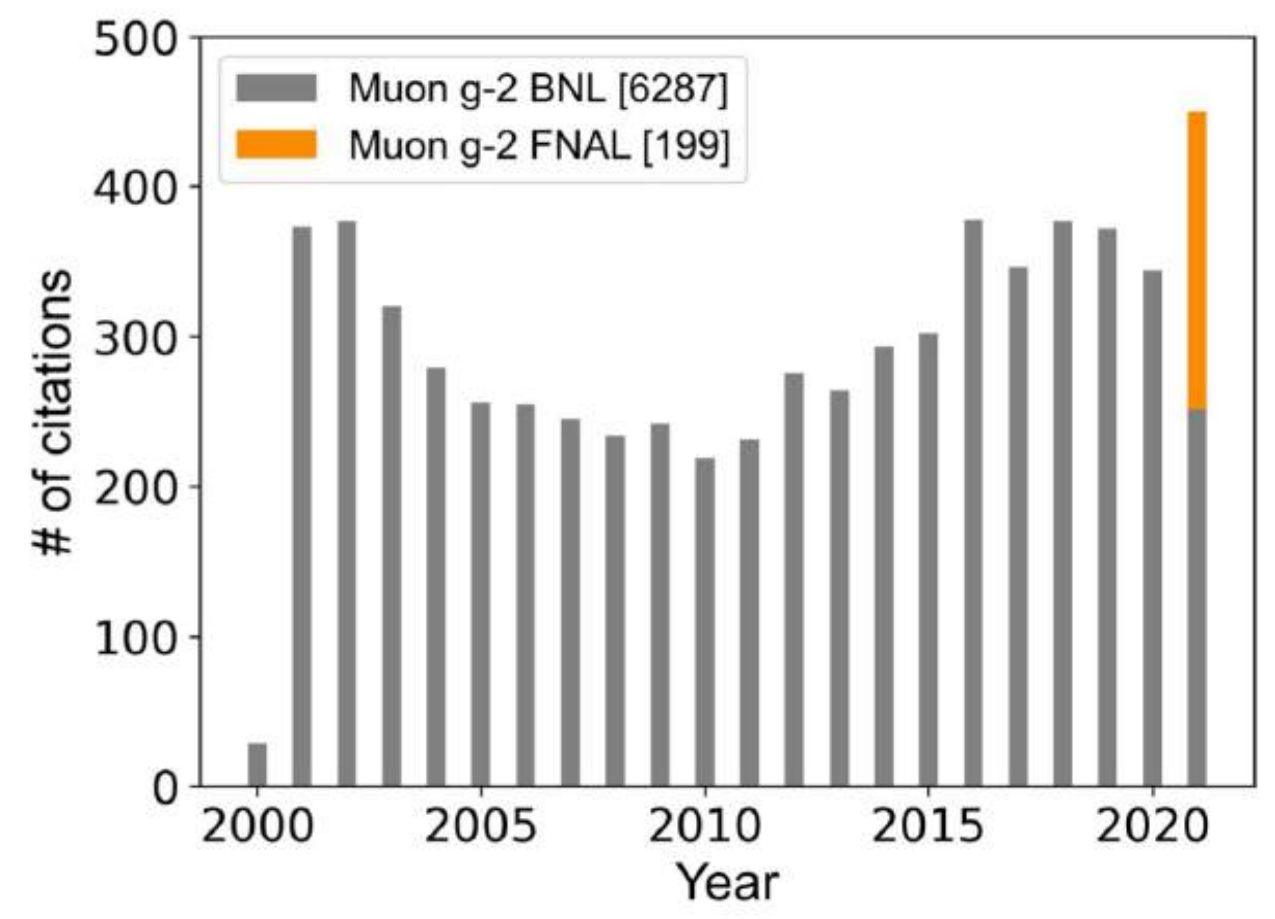




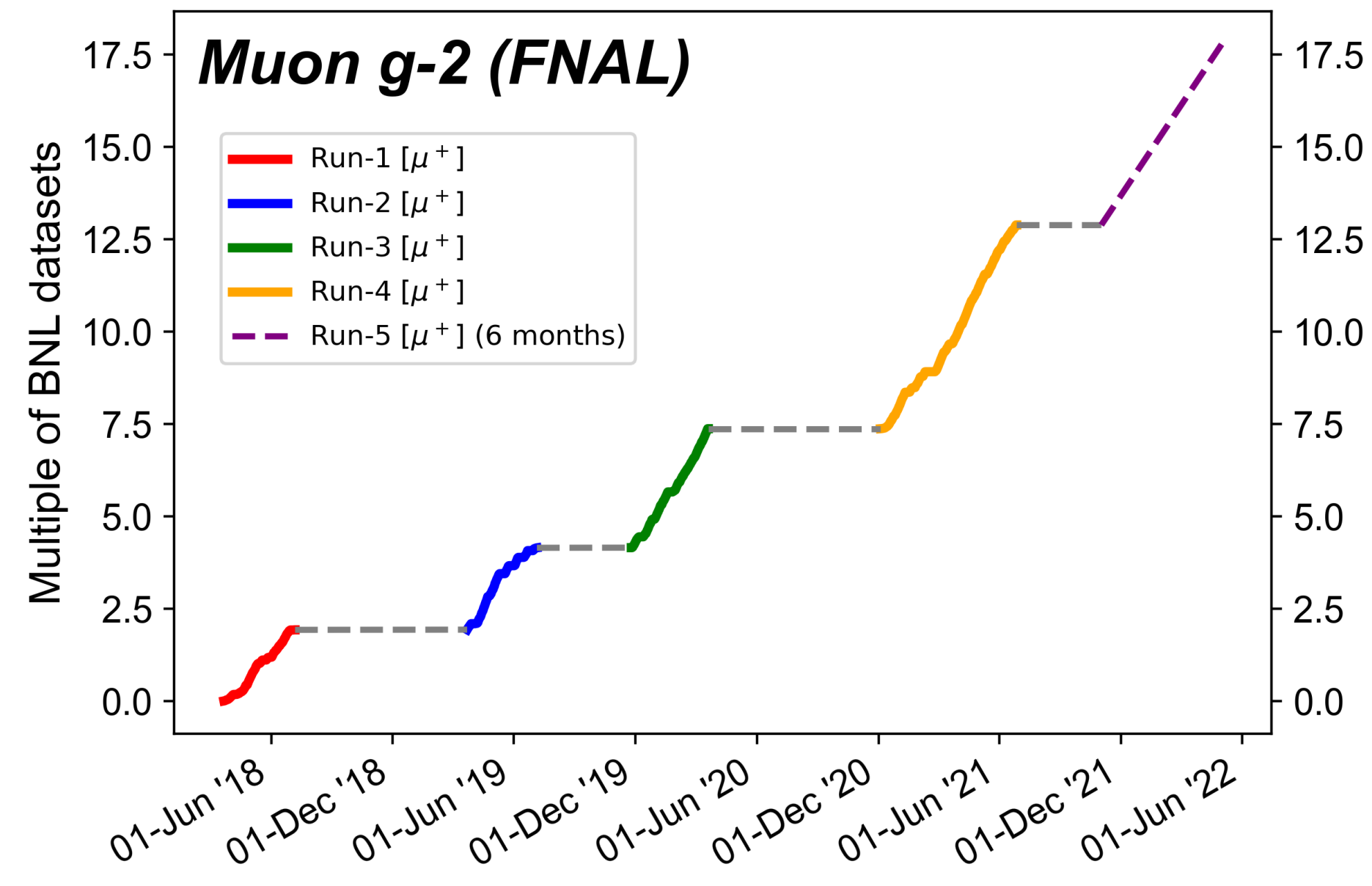

Currently at $\sim 12.43 \times$ BNL data 


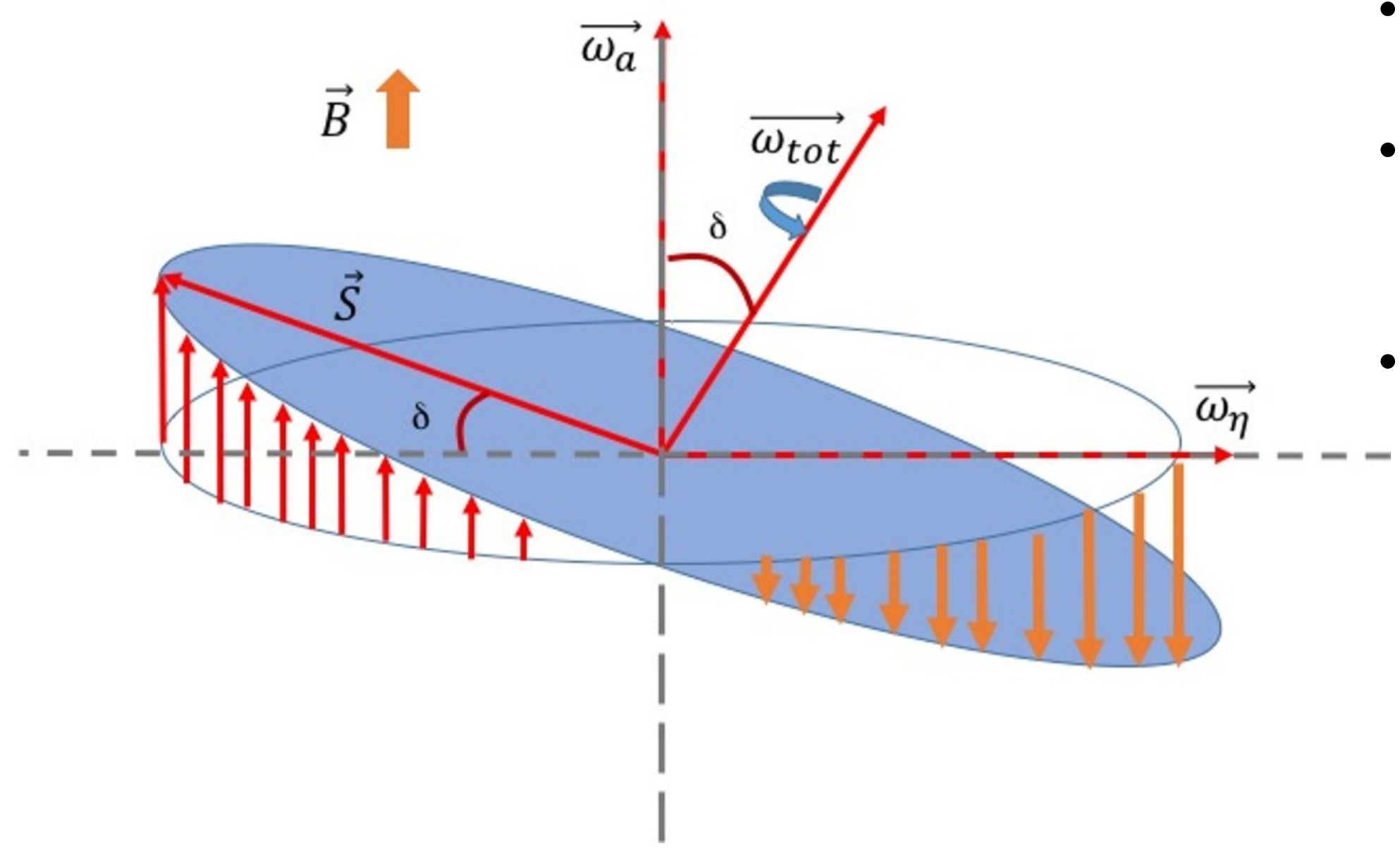

- Currently measuring $\mu^{+}$ anomalous MDM

- Measure $\mu^{+}$EDM using vertical phase asymmetry detection in calorimeters

- Measure $\mu^{-}$by reconfiguring the beamlines and storage ring (switching electric field direction)

- No other proposed experiment can do $\mu^{-}$ (JPARC $\mu^{+}$only) 
$>$ The first $a_{\mu}$ result was released (Run-1), with precision 460 ppb $>$ The combined FNAL+BNL result has a $4.2 \sigma$ tension with the SM prediction

$>$ We already have $\times 10$ more data compared to Run-1

$>$ Run-2 and Run-3 results are expected to be ready for release in $\sim 1$ year

$>$ The experiment continues physics runs to accumulate statistics for a total uncertainty of $140 \mathrm{ppb}$

$>$ Run-4 is complete, and Run-5 will begin soon 
- This material is based upon work supported by the U.S. Department of Energy, Office of Science, under Contract No. DE-FG02-08ER41546 and Contract No. DE-SC0018636.

- This document was prepared by the Muon g-2 collaboration using the resources of the Fermi National Accelerator Laboratory (Fermilab), a U.S. Department of Energy, Office of Science, HEP User Facility. Fermilab is managed by Fermi Research Alliance, LLC (FRA), acting under Contract No. DE-AC02-07CH11359.

- This research used resources of the National Energy Research Scientific Computing Center (NERSC), a U.S. Department of Energy Office of Science User Facility operated under Contract No. DE-AC02-05CH11231.

- This research was done using resources provided by the Open Science Grid, which is supported by the National Science Foundation award 1148698, and the U.S. Department of Energy's Office of Science. 


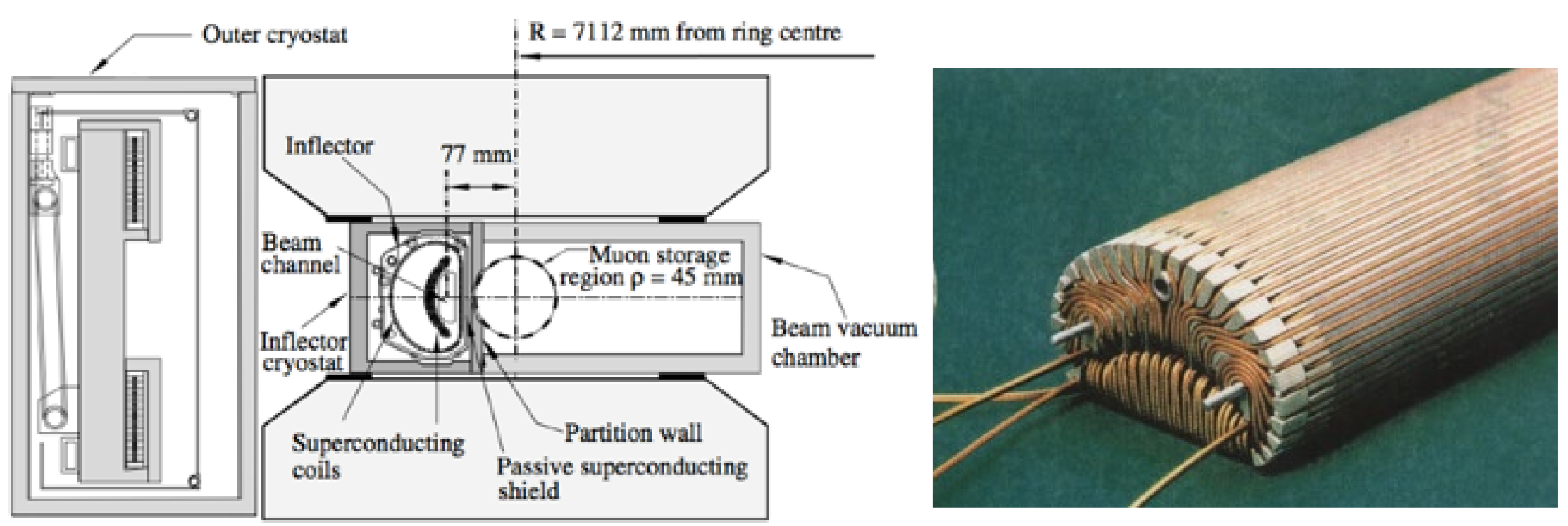

- $1.45 \mathrm{~T}$ bucking field to cancel main field

- Can't perturb main field by more than $\sim 1 \mathrm{ppm}$

- Interface optics of storage ring and the M5 beamline 


$$
\begin{aligned}
& N=N_{0} \Lambda N_{c b o} N_{2 c b o} N_{v w} e^{-t / \tau}\left(1-A A_{c b o} \cos \left(\omega_{a} t+\phi \phi_{c b o}\right)\right) \\
& N_{c b o}=1-A_{1 c b o} e^{-\frac{t}{\tau_{c b o}}} \cos \left(\omega_{c b o} t+\phi_{1 c b o}\right) \\
& N_{2 c b o}=1-A_{2 c b o} e^{-\frac{2 t}{\tau_{c b o}}} \cos \left(2 \omega_{c b o} t+\phi_{2 c b o}\right) \quad \chi^{2}=\sum_{i=1}^{n d f}\left[\frac{N_{b i n}-N_{f i t}}{\sigma\left(N_{b i n}\right)}\right]^{2} \\
& N_{v w}=1-A_{v w} e^{-\frac{t}{\tau_{v w}}} \cos \left(\omega_{v w} t+\phi_{v w}\right) \\
& A_{c b o}=1-A_{A c b o} e^{-\frac{t}{\tau_{c b o}}} \cos \left(\omega_{c b o} t+\phi_{\text {Acbo }}\right) \\
& \phi_{c b o}=1-A_{\phi c b o} e^{-\frac{t}{\tau_{c b o}}} \cos \left(\omega_{c b o} t+\phi_{\phi c b o}\right) \\
& \omega_{\text {cbo }}=\omega_{0}\left(1+2.875 e^{-\frac{t}{76}} / \omega_{0} t+5.47 e^{-\frac{t}{8.85}} / \omega_{0} t\right) \\
& \Lambda=1-K_{\text {loss }} \int L\left(t^{\prime}\right) e^{t^{\prime} / 64.4} d t
\end{aligned}
$$




\section{Straw Tracking Detectors}

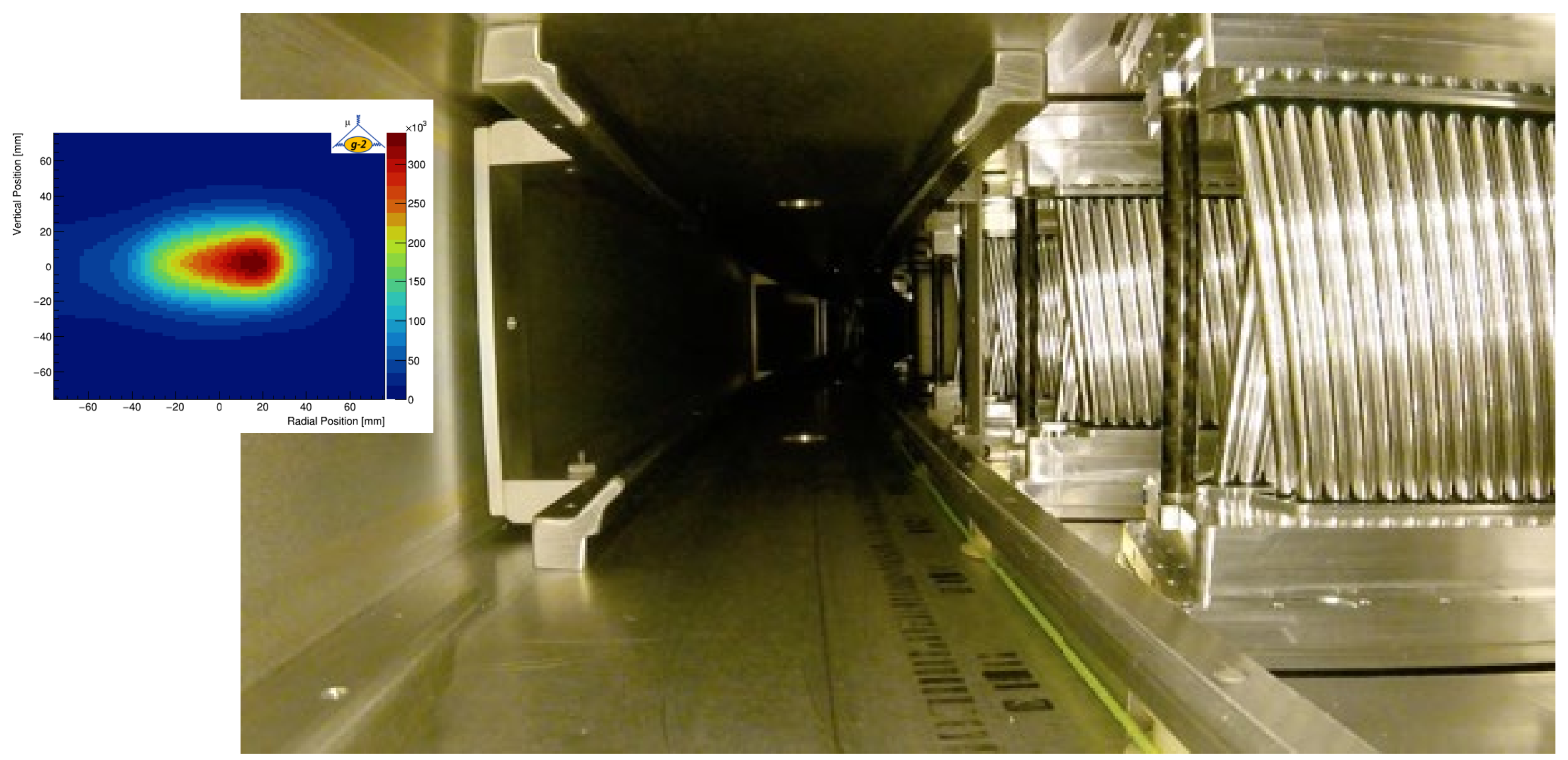

